\title{
Influencia de los principales modos anulares hemisféricos y EI Niño-Oscilación del Sur (ENOS) en las fuentes de humedad globales de Mesoamérica
}

\section{Influence of the main Annular Hemispheric Modes and ENSO on Mesoamerica's global moisture sources}

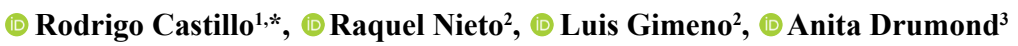 \\ ${ }^{1}$ Escuela de Física y Centro de Investigaciones Geofísicas, Universidad de Costa Rica, San José, Costa Rica \\ ${ }^{2}$ Environmental Physics Laboratory, Facultade de Ciencias, Universidade de Vigo, Ourense, España \\ ${ }^{3}$ Instituto de Ciências Ambientais, Químicas e Farmacêuticas, Universidade Federal de São Paulo, Diadema, Brazil
}

\begin{abstract}
Resumen
Se estudió la influencia de las oscilaciones atmosféricas interanuales de gran escala en la captación y el transporte de humedad proveniente de las principales fuentes oceánicas con impacto en Mesoamérica utilizando el modelo lagrangiano FLEXPART alimentado con los datos del reanálisis ERA-Interim (1979-2012) para evidenciar cómo los cambios en los sumideros de humedad asociados con cada una de estas fuentes de evaporación reproducen los ya conocidos patrones de variación de los sistemas atmosféricos y de precipitación regional de El Niño-Oscilación del Sur y de la Oscilación Ártica. El hallazgo más importante, que no se había registrado antes para la región de Mesoamérica, es la teleconexión con la Oscilación Antártica, lo que revela una correlación estadística más significativa con el transporte de humedad proveniente del Pacífico durante la estación seca y una un poco más débil durante la estación lluviosa. Estos resultados pueden emplearse como predictores del comportamiento de la distribución de la precipitación, especialmente en el momento de su inicio y en el de su primer máximo.
\end{abstract}

Palabras clave: Oscilación Antártica (AAO); Oscilación Ártica (AO); ENOS; Mesoamérica; transporte de humedad; FLEXPART.

\begin{abstract}
The influence of the interannual large-scale atmospheric oscillations in the uptake and moisture transport from the major oceanic moisture sources with impact over Mesoamerica is investigated herein using a sophisticated Lagrangian approach informed by ERA-Interim reanalysis data (1979-2012), which showed how the moisture sinks changes associated with the evaporation sources reproduce the well-known atmospheric systems variation patterns and the regional precipitation linked to El Niño-Southern Oscillation and the Arctic Oscillation. However, the most important result, not revealed before for the Mesoamerican region, is the Antarctic Oscillation teleconnection, which highlights a stronger statistical correlation with the Pacific moisture transport during the dry season and a little weaker correlation during the rainy season. These results may be used as predictors for the precipitation distribution behavior, mainly during its onset period and for its first maximum.
\end{abstract}

Keywords: Antarctic Oscillation (AAO); Artic Oscillation (AO); ENSO; Mesoamerica; moisture transport; FLEXPART.

\section{Introducción}

La región de estudio es la denominada región climática continental de América Central (CAM), la cual fue identificada como punto caliente del cambio climático por Giorgi \& Francisco (2000) y está circunscrita por las coordenadas $10^{\circ} \mathrm{N}-30^{\circ} \mathrm{N}$ de latitud y $116^{\circ} \mathrm{O}-83^{\circ} \mathrm{O}$ de longitud (Figura 1 , mapa superior). Dicha región se utiliza ampliamente para los informes del Panel Intergubernamental sobre Cambio Climático (Nieto, et al., 2014a, Barker, et al., 2007). En el presente estudio, sin embargo, se la denomina región climática continental de Mesoamérica (MAM), ya que se incluyó el territorio de México.

\footnotetext{
*Correspondencia:

Rodrigo Castillo; rodrigo.castillorodriguez@ucr.ac.cr

Recibido: 21 de marzo de 2019

Aceptado: 30 de octubre de 2019

Editor: José Daniel Pabón Caicedo
} 


\section{Región Climática [MAM]}
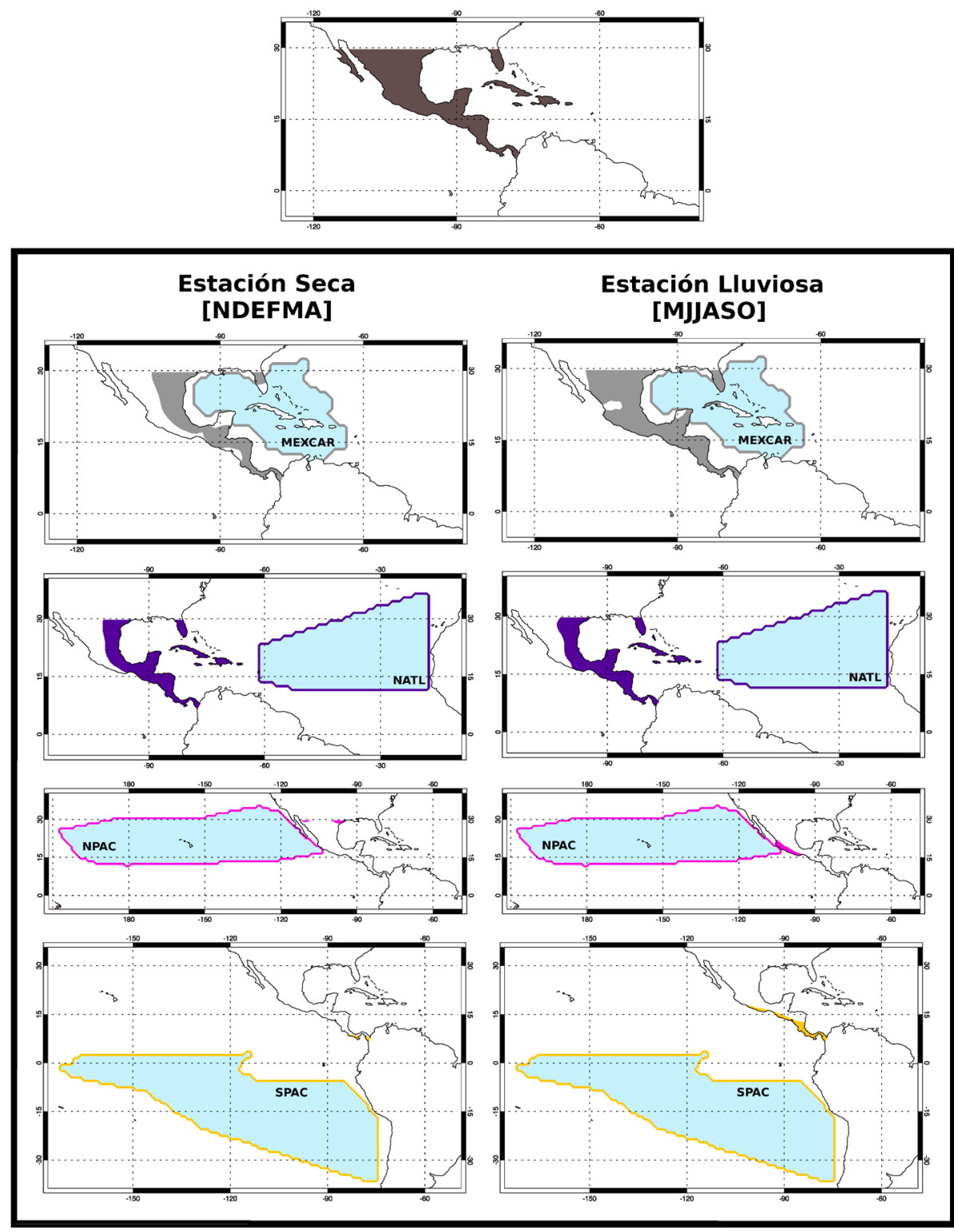

Figura 1. Representación esquemática estacional de la magnitud de los sumideros de humedad provenientes de las principales fuentes oceánicas que afectaron la región climática continental MAM (mapa superior) para el periodo de 1979 a 2012. Solo los valores $(|\mathrm{E}-\mathrm{P}<0|$ ) por encima de $0,05 \mathrm{~mm} /$ día se graficaron y se muestran con el mismo color que su respectiva fuente oceánica

La precipitación de una determinada región proviene de la evaporación y la evapotranspiración de regiones continentales, por ejemplo, las selvas tropicales, o de la advección de humedad procedente de regiones oceánicas, cuyo papel en la alimentación de la rama terrestre del ciclo hidrológico es insustituible. El transporte de la humedad a escala global proveniente de las principales fuentes oceánicas ha sido estudiado por Gimeno, et al. (2010), quienes caracterizaron doce principales fuentes globales de humedad, de las cuales cuatro tienen impacto en la región MAM: Pacífico Norte, Pacífico Sur, Atlántico Norte y Golfo de México - Mar Caribe (Figura 2).
Si bien las regiones oceánicas determinadas por Gimeno, et al. (2010) son las principales fuentes de humedad a escala global, el transporte de humedad desde estas fuentes puede presentar cambios asociados con los principales modos de variabilidad atmosférica interanual: El Niño-Oscilación del Sur (ENOS), la Oscilación Ártica (Arctic Oscillation, AO) y la Oscilación Antártica (Antarctic Oscillation, AAO). Por esta razón, Castillo, et al. (2014a) utilizaron un enfoque lagrangiano para analizar la variabilidad climática del transporte de humedad desde las principales fuentes oceánicas de escala planetaria para ENOS, en tanto que Nieto, et al. (2014b) la analizaron a partir de los modos anulares 


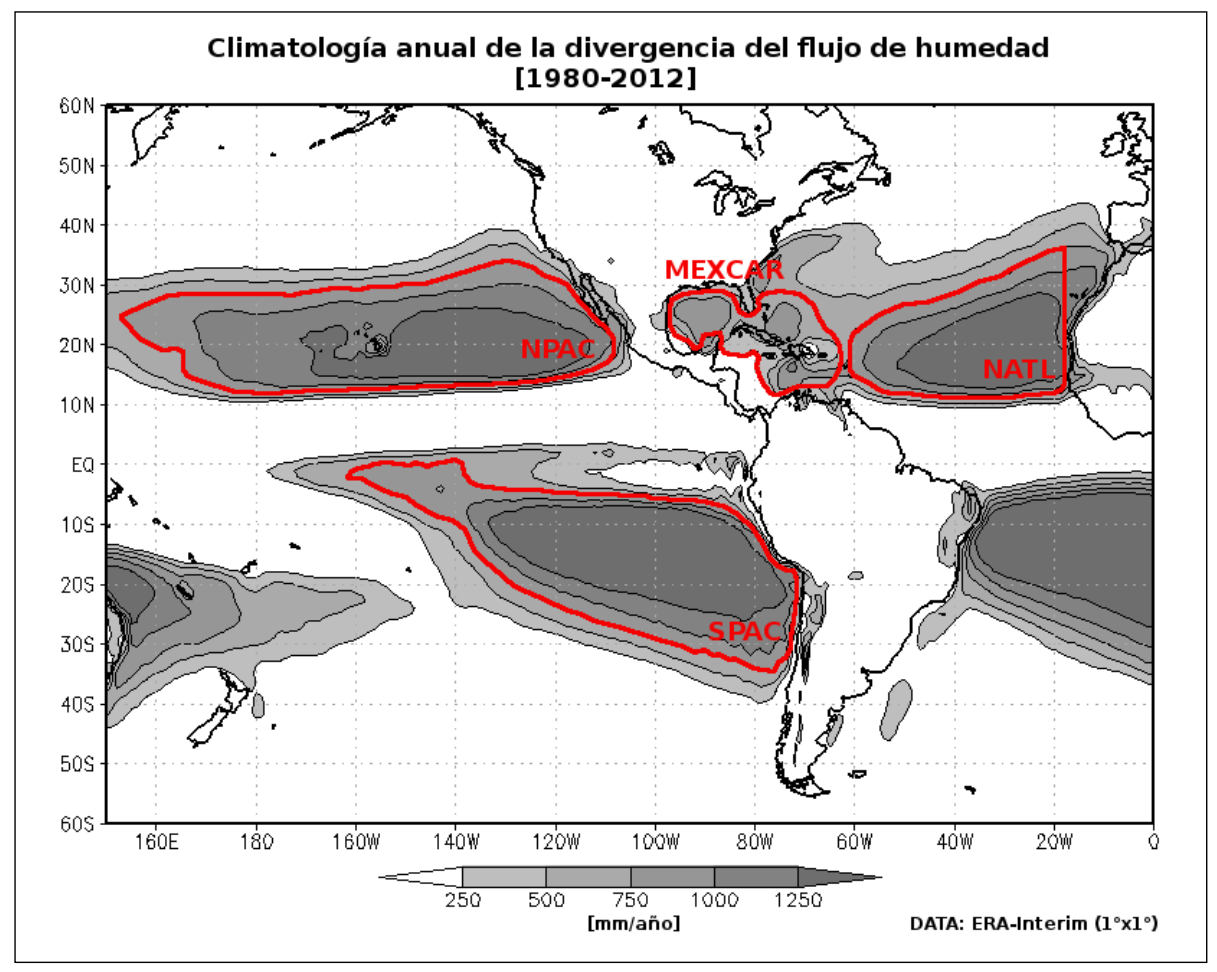

Figura 2. Principales fuentes oceánicas de humedad que afectaron la región climática continental MAM: climatología anual de la divergencia del integrado vertical del flujo de humedad (mm/año) para el periodo de 1980 a 2012. Las líneas rojas indican el umbral de 750 mm/año, el cual define las fuentes de humedad oceánicas (Gimeno, et al., 2010). Según ese criterio, las cuatro fuentes oceánicas analizadas fueron la del Pacífico Norte (NPAC), el Pacífico Sur (SPAC), el Atlántico Norte (NATL) y el Golfo de México - Mar Caribe (MEXCAR)

hemisféricos. Aunque la metodología para la selección de las composiciones difiere en estos dos trabajos, en ambos se evidenció cómo los cambios en los sumideros de humedad asociados con cada una de estas fuentes de evaporación reproducen los ya conocidos patrones de variabilidad atmosférica y de precipitación a gran escala, aunque en ninguno de estos trabajos se abordó el análisis de una región específica como sumidero de humedad.

En este contexto, y con el fin de examinar la influencia que tienen las oscilaciones atmosféricas de escala global e interanual ENOS, AO y AAO en la captación y el transporte de humedad semestral proveniente de las principales fuentes oceánicas que tienen impacto en la región MAM, se usó el modelo lagrangiano FLEXPART (Stohl, et al., 1998; 2005) alimentado con datos del reanálisis ERA-Interim (Dee, et al., 2011) para el periodo de 1979 a 2012.

El tratamiento semestral estacional responde a que la mayor parte de la región MAM tiene dos estaciones bien definidas, una seca, de noviembre a abril, y otra lluviosa, de mayo a octubre. Este comportamiento se debe principalmente a una combinación de sistemas que involucran la migración latitudinal de la zona de convergencia intertropical (Intertropical Convergence Zone, ITCZ), la variación estacional de la radiación solar, que influye en el flujo de calor latente, y los vientos de bajo nivel que interactúan con la orografía local (Alfaro, et al., 2018).
La temporada de lluvias en la parte centro-sur de México, la mayor parte de América Central, y ciertas zonas del Caribe, se caracteriza por una distribución bimodal de las precipitaciones, con máximos entre mayo y junio y septiembre y octubre y un mínimo relativo en julio y agosto (Mosiño \& García, 1966; Coen, 1973; Magaña, et al., 1999). Este mínimo relativo de la precipitación se conoce como la "sequía" de verano, "canícula" o "veranillo", dependiendo de la región en la que ocurra (midsummer drought, MSD) (Karnauskas, et al., 2013). Sin embargo, cabe destacar que la parte norte de México, que incluye la Sierra Madre Oriental y la Occidental, no tiene este mismo patrón y se rige, en cambio, por la dinámica del monzón de América del Norte, el cual se ve influenciado por los aumentos en el contenido de humedad en la baja tropósfera de la piscina de agua cálida del hemisferio occidental y el papel del chorro de bajo nivel del Caribe (Caribbean Low Level Jet, CLLJ) (Amador, 2008) como transportador de humedad para esta región (Wang \& Enfield, 2001, 2003; Durán-Quesada, et al., 2017a).

Recientemente, Durán-Quesada, et al. (2017b) emplearon la metodología lagrangiana en fuentes de humedad regionales para analizar el papel modulador de ENOS en el suministro de humedad que alimenta la precipitación en la región de América Central, el cual ya había sido establecido por Maldonado, et al. (2016) como el principal modo de 
variabilidad interanual en el control de las características del MSD a través del patrón de dipolo de la temperatura superficial del mar (sea surface temperature, SST) en las aguas vecinas a Centroamérica. Sin embargo, a pesar de que los trabajos de Maldonado, et al. (2016) y Durán-Quesada, et al. (2017b) evidencian que ENOS es el principal modo de variabilidad del control de las variaciones interanuales de las características atmosféricas regionales mediante la SST, no ha habido estudios que analicen si esa variabilidad puede estar relacionada con los modos anulares hemisféricos.

Por todo lo expuesto, en este trabajo se propuso hacer un análisis unificado de las oscilaciones atmosféricas de escala global e interanual guiado por un mismo criterio de selección de los años incluidos en las composiciones de las diferentes fases de las oscilaciones en estudio con base en los periodos semestrales (seco y lluvioso), los cuales responden a los mecanismos atmosféricos prevalentes en la mayor parte del territorio mesoamericano, subsanando así las discrepancias metodológicas encontradas en los trabajos de Castillo, et al. (2014a) y Nieto, et al. (2014b), e incorporando la pregunta de si hay variabilidad interanual relacionada con la $\mathrm{AO}$ y la $\mathrm{AAO}$.

\section{Datos y metodología}

En la Figura 2 se aprecian las principales fuentes de humedad oceánica que afectan a la región continental MAM, las cuales se determinaron siguiendo los mismos criterios de Gimeno, et al. (2010), pero considerando un periodo de 33 años. Las cuatro principales fuentes de humedad oceánica consideradas fueron: Pacífico Norte (NPAC), Pacífico Sur (SPAC), Atlántico Norte (NATL) y Golfo de México - Mar Caribe (MEXCAR).

Para analizar la transferencia del contenido de humedad en el aire entre lugares, se utilizó la integral vertical del flujo de vapor de agua, la cual proporciona una aproximación al transporte de humedad en la atmósfera y se define como:

$$
\Theta=\frac{1}{g} \int_{0}^{p_{s}} q \vec{V} d p
$$

donde $g$ es la aceleración debida a la gravedad, $q$ es la humedad específica, $p_{s}$ es la presión de la superficie y $\vec{V}$ es el vector del viento horizontal. Teniendo en cuenta la ecuación de conservación de la masa, se puede describir el balance hidrológico en la atmósfera mediante la siguiente relación:

$$
\frac{\partial W}{\partial t}+\nabla \cdot \Theta=E-P
$$

donde $W$ es el agua precipitable dada por:

$$
W=\frac{1}{g} \int_{0}^{p_{s}} q d p
$$

En escalas temporales grandes como las consideradas en este estudio, la variación temporal del contenido de agua precipitable en la columna vertical es despreciable, por lo que la integral vertical del transporte de humedad será equivalente a la evaporación neta $(E)$ menos la precipitación neta $(P)$ (Trenberth \& Guillemot, 1998), como se muestra a continuación:

$$
\nabla \cdot \Theta=E-P
$$

Para realizar este estudio se empleó la metodología desarrollada por Stohl \& James (2004, 2005) y el modelo lagrangiano de dispersión de partículas FLEXPART, versión 9.0 (Stohl, et al., 1998, 2005), inicializado con datos del reanálisis ERA-Interim (Dee, et al., 2011) para un periodo de 33 años (noviembre de 1979 a octubre de 2012). Se recurrió a este conjunto de datos porque se ha demostrado que el rendimiento del ERA-Interim en la reproducción del ciclo hidrológico y en términos de cierre del balance hídrico es mucho mejor que el del ERA-40 (Trenberth, et al., 2011), y que el de productos más recientes de reanálisis como el MERRA y el CFSR (Lorenz \& Kunstmann, 2012). Además, desde sus inicios la evaluación espacial del modelo FLEXPART se ha hecho con datos provenientes del European Centre for Medium-Range Weather Forecasts (ECMWF) (Stohl, et al., 1998), lo que le otorga mayor confiabilidad.

Los datos de los años anteriores a 1979 no se utilizaron para ejecutar el modelo FLEXPART, ya que los resultados del reanálisis anteriores a la inclusión de los datos de satélite se consideran de insuficiente calidad (especialmente los referentes a los océanos) (Bengtsson, et al., 2004), principalmente por dos razones: (i) el modelo FLEXPART utiliza variables derivadas como datos de entrada, por ejemplo, el viento zonal $(u)$, el viento meridional $(v)$ y la humedad específica $(q)$, los cuales no son fiables antes de esta fecha, pues son muy propensos a errores, lo que se refleja en los resultados del modelo (Stohl, et al., 2005), y (ii) es imposible trabajar con datos obtenidos antes de la incorporación de las imágenes de satélite en el reanálisis a partir de 1979. Antes de esta fecha, no había suficientes observaciones sobre las grandes áreas oceánicas y los conjuntos de datos son mucho menos confiables (Bengtsson, et al., 2004; Uppala, et al., 2005).

Los modelos lagrangianos calculan las trayectorias de un gran número de pequeñas parcelas infinitesimales de aire (denominadas "partículas") para modelar el transporte y la difusión de trazadores atmosféricos (Stohl, et al., 2005). Al inicio de la ejecución del modelo, la atmósfera fue "rellenada" de manera homogénea con partículas de igual masa, cada una de las cuales representa una fracción de la masa atmosférica total (Stohl \& James, 2004, 2005). Durante la ejecución, estas partículas son advectadas usando el campo de velocidad tridimensional del reanálisis, con movimientos turbulentos estocásticos y convectivos superpuestos. En la capa atmosférica límite (planetary boundary layer, PBL), estos movimientos aleatorios se calcularon mediante la solución de las ecuaciones de Langevin para la turbulencia gaussiana (Stohl \& Thomson, 1999). Estas ecuaciones usan escalas de tiempo lagrangiano y desviaciones estándar 
para las componentes de viento, que se calculan utilizando los parámetros de la PBL del ECMWF (Hanna, 1984). La altura de la PBL se determina mediante una combinación del número de Richardson y la técnica de elevación de la parcela (Vogelezang \& Holtslag, 1996); se asume que la turbulencia por fuera de la PBL es muy pequeña. Además, el conjunto de datos globales no resuelve las células convectivas individuales, sino que reproduce los efectos de convección a gran escala.

Las posiciones de las partículas y la humedad específica $(q)$ se registran cada seis horas. Así, los aumentos (evaporación, $e$ ) y disminuciones (precipitación, $p$ ) en la humedad de la partícula a lo largo de la trayectoria se calculan a partir de cambios en la humedad específica $(q)$ con respecto al tiempo, como lo muestra la ecuación 5:

$$
e-p=m \frac{d q}{d t}
$$

donde $m$ es la masa de cada partícula.

La suma de los cambios de humedad $(e-p)$ de todas las partículas en la columna atmosférica sobre un área específica, $A$, genera el flujo de agua dulce superficial $(E-P)$, donde $E$ es la tasa de evaporación por unidad de área y $P$ es la tasa de precipitación por unidad de área (ecuación 6):

$$
E-P \approx \frac{\sum_{k=1}^{K}(e-p)_{k}}{A}
$$

donde $K$ es el número total de partículas en la columna atmosférica.

En este trabajo, la atmósfera global se dividió en alrededor de 2 millones de partículas. Cada partícula se rastreó durante 10 días, ya que este es el tiempo medio de residencia del vapor de agua en la atmósfera (Numaguti, 1999). Las trayectorias se calcularon utilizando los datos disponibles del reanálisis con intervalos de seis horas $(00,06,12$ y 18 UTC) y con una resolución espacial de $1^{\circ}$ de latitud por $1^{\circ}$ de longitud. Se utilizaron los 61 niveles verticales de los datos del reanálisis, de 0,1 a $1.000 \mathrm{hPa}$, con aproximadamente 14 niveles por debajo de $1.500 \mathrm{~m}$ y 23 entre $1.500 \mathrm{~m}$ y 5.000 $\mathrm{m}$. Estas partículas se rastrearon hacia adelante en el tiempo en las cuatro regiones oceánicas de estudio para calcular el campo $(E-P)$ cada seis horas durante los diez días de transporte y promediar para obtener el campo diario usado para establecer el campo semestral que supera la sensibilidad computacional debida al periodo de tiempo empleado, como se explica más detalladamente en Castillo, et al. (2014b).

El análisis del papel de los patrones de teleconexión climática en la variabilidad de las fuentes oceánicas que afectan los sumideros de humedad de la región climática continental MAM, se hace con la técnica de composiciones. Esta técnica, representada en la ecuación 7, es una herramienta muy conveniente para construir la estimación del estado promedio de una variable condicionada por el valor de un índice externo.

$$
\left\langle X_{\Gamma}\right\rangle=\frac{1}{i} \sum_{j=1}^{i} X_{t j}
$$

donde $\Gamma$ es el compuesto del campo $X$ condicionado por un índice $t$ para un número de observaciones $i$.

Estas composiciones se hicieron para los campos de los sumideros de humedad $(E-P<0)$ resultantes de las salidas de FLEXPART, los cuales son válidos en la escala de tiempo de este análisis (Castillo, et al., 2014b), y considerando los años de las distintas fases (positivas, negativas y neutrales) de las oscilaciones atmosféricas de escala global e interanual.

Para el caso de ENOS, se utilizó el índice oceánico del Niño (Oceanic Niño Index, ONI) en la región de El Niño $3.4\left(5^{\circ} \mathrm{N}-5^{\circ} \mathrm{S}, 120^{\circ}-170^{\circ} \mathrm{W}\right)$, tomado del National Oceanic and Atmospheric Administration/Climate Prediction Center (NOAA/CPC) (Smith, et al., 2008), el cual se basa en el promedio trimestral de las anomalías de la SST calculadas con la base de datos ERSSTv5 (Huang, et al., 2017). Este índice es la principal medida oceánica para el monitoreo, evaluación y predicción de ENOS. En este caso, se hicieron promedios del índice en las estaciones secas (noviembre a abril, NDEFMA) y lluviosas (mayo a octubre, MJJASO) (Tabla 1); luego se determinaron los seis episodios de mayor intensidad de las fases positiva y negativa por estación utilizando los valores umbrales del índice en los percentiles 18 y 82 como se muestra en la Tabla 2, en tanto que para la fase neutral se seleccionaron los años con los índices más cercanos a cero. Se obtuvo, así, la composición de la fase de El Niño (1983, 1987, 1992, 1995, 1998, 2010), la fase de La Niña $(1985,1989,1999,2000,2008,2011)$ y la fase neutra (1981, 1982, 1990, 1993, 1994, 2002) para la estación seca, y la composición de la fase de El Niño (1982, 1987, 1991, 1997, 2002, 2009), de la fase de La Niña $(1988,1998,1999$, $2007,2010,2011)$ y de la fase neutra $(1980,1983,2001$, 2003, 2005, 2012) para la estación lluviosa.

Para el índice de la Oscilación Ártica (Arctic Oscillation Index, AOI) y el de la Oscilación Antártica (Antarctic Oscillation Index, AAOI), se siguió el esquema planteado por Nieto, et al. (2014b) en la selección de las composiciones. Entre las diferentes metodologías comúnmente utilizadas para determinar los eventos extremos de la $\mathrm{AO}$ y de la AAO, se pueden citar: (i) las que se formulan a partir del análisis de componentes principales (principal components, PC) de alguna variable meteorológica en los extratrópicos (por ejemplo, la altura geopotencial, la presión media a nivel del mar, el viento o la temperatura) (Thompson \& Wallace, 2000; Nan \& Li, 2003); (ii) las que calculan la diferencia entre la presión media zonal normalizada entre dos latitudes usando datos de reanálisis (Gong \& Wang, 1999; Li \& Wang, 2003), o entre dos puntos seleccionados (Hurrell, 1995), y (iii) las que se basan en datos obtenidos de estaciones de observación (Marshall, 2003; Visbeck, 2009). Según Ho, et al. (2012), hay algunas inconsistencias con respecto a la determinación de los episodios extremos de 
ambas oscilaciones, especialmente de la AAO, que se deben principalmente a las diferentes metodologías y conjuntos de datos aplicados en la definición de los índices. Para el AAOI, pero también para el AOI (Ho, et al., 2012), se evidenció que los índices calculados con datos de reanálisis son más efectivos cuando se intenta comprender la relación con sus impactos. Sin embargo, antes de la incorporación de los

Tabla 1. Índices estacionales considerados para las composiciones de las fases de El Niño-Oscilación del Sur para el periodo de 1979 a 2012

\begin{tabular}{|c|c|c|c|}
\hline & \multicolumn{2}{|c|}{ ONI } & \\
\hline & Seca & Lluviosa & \\
\hline 1980 & 0,48 & $0,20(*)$ & $1980(*)$ \\
\hline $1981(*)$ & $-0,27(*)$ & $-0,23$ & 1981 \\
\hline $1982(*)$ & $0,08(*)$ & $1,15(+)$ & $1982(+)$ \\
\hline $1983(+)$ & $1,88(+)$ & $0,12(*)$ & $1983(*)$ \\
\hline 1984 & $-0,60$ & $-0,37$ & 1984 \\
\hline 1985(-) & $-0,90(-)$ & $-0,52$ & 1985 \\
\hline 1986 & $-0,37$ & 0,35 & 1986 \\
\hline $1987(+)$ & $1,12(+)$ & $1,42(+)$ & $1987(+)$ \\
\hline 1988 & 0,58 & $-1,22(-)$ & 1988(-) \\
\hline 1989(-) & $-1,43(-)$ & $-0,33$ & 1989 \\
\hline $1990(*)$ & $0,10(*)$ & 0,33 & 1990 \\
\hline 1991 & 0,33 & $0,63(+)$ & 1991(+) \\
\hline $1992(+)$ & $1,47(+)$ & 0,33 & 1992 \\
\hline $1993(*)$ & $0,20(*)$ & 0,37 & 1993 \\
\hline $1994(*)$ & $0,13(*)$ & 0,48 & 1994 \\
\hline $1995(+)$ & $0,77(+)$ & $-0,40$ & 1995 \\
\hline 1996 & $-0,78$ & $-0,33$ & 1996 \\
\hline 1997 & $-0,27$ & $1,65(+)$ & $1997(+)$ \\
\hline 1998(+) & $1,88(+)$ & $-0,70(-)$ & 1998(-) \\
\hline 1999(-) & $-1,33(-)$ & $-1,12(-)$ & 1999(-) \\
\hline $2000(-)$ & $-1,37(-)$ & $-0,58$ & 2000 \\
\hline 2001 & $-0,55$ & $-0,18(*)$ & 2001(*) \\
\hline $2002(*)$ & $-0,07(*)$ & $\mathbf{0 , 8 3}(+)$ & $2002(+)$ \\
\hline 2003 & 0,72 & $0,07(*)$ & $2003(*)$ \\
\hline 2004 & 0,32 & 0,50 & 2004 \\
\hline 2005 & 0,57 & $-0,03(*)$ & $2005(*)$ \\
\hline 2006 & $-0,62$ & 0,27 & 2006 \\
\hline 2007 & 0,43 & $-0,75(-)$ & 2007(-) \\
\hline 2008(-) & $-1,37(-)$ & $-0,45$ & 2008 \\
\hline 2009 & $-0,58$ & $0,53(+)$ & $2009(+)$ \\
\hline $2010(+)$ & $1,17(+)$ & $-1,07(-)$ & 2010(-) \\
\hline 2011(-) & $-1,20(-)$ & $-0,68(-)$ & 2011(-) \\
\hline 2012 & $-0,73$ & $0,17(*)$ & 2012(*) \\
\hline
\end{tabular}

ONI: Oceanic Niño Index; (+): Niño; (*): Neutro; (-): Niña datos satelitales (los anteriores a 1979), es probable que los índices basados en el reanálisis no sean confiables, y es mejor elegir aquellos provenientes de estaciones de observación. Tomando esto en consideración, como lo hicieron Nieto, et al. (2014b), se valoraron dos índices para cada oscilación con base en diferentes metodologías que usan datos de la presión a nivel del mar normalizada: uno basado en estaciones de observación y otro basado en reanálisis.

En cuanto al AOI basado en los datos de las estaciones de observación, dado que muchos autores (Thompson \& Wallace, 2001) consideran que la Oscilación del Atlántico Norte (North Atlantic Oscillation, NAO) es solo la manifestación de la $\mathrm{AO}$ en la región atlántica, se usó el índice basado en la diferencia de presión a nivel del mar normalizada entre Lisboa, Portugal, y Stykkisholmur-Reykjavik, Islandia (Hurrell, 1995) (https://climatedataguide.ucar.edu/ climate-data/hurrell-north-atlantic-oscillation-nao-indexstation-based). En tanto que para el AOI basado en los reanálisis (National Centers for Environmental Prediction/ National Center for Atmospheric Research, NCEP/NCAR) (Kalnay, et al., 1996), se usó el índice definido como la diferencia mensual normalizada de la presión media zonal a nivel del mar entre $\operatorname{los} 35^{\circ} \mathrm{N}$ y los $65^{\circ} \mathrm{N}$ (Li \& Wang, 2003) (http://ljp.gcess.cn/dct/page/65569). Al igual que con el ONI, se hicieron los promedios estacionales para ambos índices (Tabla 3) estableciendo los seis eventos extremos de cada fase por estación con los valores de umbral de la Tabla 2, y para la fase neutral se seleccionaron los años con los índices más próximos a cero. En la estación seca se obtuvieron las siguientes composiciones: $\mathrm{AO}(+)(1989$, 1990, 1993, 2000, 2007, 2012), AO(-) (1984, 1986, 1996, 2006, 2010, 2011), y en la fase neutra los siguientes:

Tabla 2. Valores umbrales de los índices de las oscilaciones atmosféricas de escala global para la selección de los episodios extremos durante el periodo de 1979 a 2012

\begin{tabular}{|c|c|c|c|c|}
\hline & \multicolumn{2}{|c|}{ ONI } & & \\
\hline Percentil & Seca & Lluviosa & & \\
\hline 18 & $-0,85$ & $-0,64$ & & \\
\hline \multirow[t]{3}{*}{82} & $-0,75$ & 0,52 & & \\
\hline & \multicolumn{4}{|c|}{ AOI } \\
\hline & Observación & Reanálisis & Observación & Reanálisis \\
\hline Percentil & \multicolumn{2}{|c|}{ Seca } & \multicolumn{2}{|c|}{ Lluviosa } \\
\hline 18 & $-0,30$ & $-0,23$ & $-0,74$ & $-0,33$ \\
\hline \multirow[t]{3}{*}{82} & 1,19 & 1,80 & 0,23 & 0,50 \\
\hline & \multicolumn{4}{|c|}{ AAOI } \\
\hline & Observación & Reanálisis & Observación & Reanálisis \\
\hline Percentil & \multicolumn{2}{|c|}{ Seca } & \multicolumn{2}{|c|}{ Lluviosa } \\
\hline 18 & $-0,59$ & $-0,11$ & $-0,62$ & $-0,13$ \\
\hline 82 & 1,05 & 1,72 & 0,98 & 2,20 \\
\hline
\end{tabular}

ONI: Oceanic Niño Index; AOI: Artic Oscillation Index; AAOI: Antarctic Oscillation Index 
Tabla 3. Índices estacionales considerados para las composiciones de las fases de la Oscilación Ártica para el periodo de 1979 a 2012.

\begin{tabular}{|c|c|c|c|c|c|}
\hline & \multicolumn{4}{|c|}{ AOI } & \\
\hline & \multicolumn{2}{|c|}{ Seca } & \multicolumn{2}{|c|}{ Lluviosa } & \\
\hline & Observación & Reanálisis & Observación & Reanálisis & \\
\hline $1980(*)$ & 0,20 & $-0,08(*)$ & $-0,70$ & $0,02(*)$ & 1980 \\
\hline 1981(*) & $-0,22$ & $-0,14(*)$ & $0,05(*)$ & $-0,51(-)$ & 1981(*) \\
\hline $1982(*)$ & $-0,02(*)$ & 0,68 & 0,22 & 0,15 & 1982 \\
\hline 1983 & 1,18 & 1,00 & 0,20 & $0,67(+)$ & $1983(+)$ \\
\hline 1984(-) & $-0,20$ & $-0,30(-)$ & $0,40(+)$ & $0,04(*)$ & $1984(+)$ \\
\hline $1985(*)$ & $-0,27$ & $0,03(*)$ & $-0,57$ & 0,11 & 1985 \\
\hline 1986(-) & $0,00(*)$ & $-0,38(-)$ & $0,03(*)$ & 0,34 & $1986(*)$ \\
\hline 1987 & 0,25 & 0,75 & $-0,97(-)$ & 0,18 & 1987 \\
\hline 1988 & $-0,38(-)$ & 1,03 & 0,18 & 0,11 & 1988 \\
\hline $1989(+)$ & $1,32(+)$ & $2,95(+)$ & $0,23(+)$ & $0,70(+)$ & $1989(+)$ \\
\hline $1990(+)$ & $1,13(+)$ & $2,69(+)$ & $-0,07\left(^{*}\right)$ & $0,66(+)$ & 1990 \\
\hline 1991 & 0,37 & 1,02 & $-0,08$ & $-0,10(*)$ & 1991(*) \\
\hline 1992 & 1,10 & 1,70 & 0,08 & $-0,24$ & 1992(*) \\
\hline 1993(+) & $1,42(+)$ & $2,32(+)$ & $-1,27(-)$ & $-0,69(-)$ & 1993(-) \\
\hline 1994 & $2,03(+)$ & 1,56 & $0,00(*)$ & $0,51(+)$ & $1994(+)$ \\
\hline 1995 & 0,90 & 1,11 & $-0,80(-)$ & $-0,06\left(^{*}\right)$ & 1995(-) \\
\hline 1996(-) & $-1,88(-)$ & $-1,97(-)$ & $0,28(+)$ & $-0,38(-)$ & 1996 \\
\hline 1997 & $-0,32(-)$ & 0,68 & $-1,10(-)$ & $-0,60(-)$ & 1997(-) \\
\hline 1998 & $0,13(*)$ & 0,78 & $-0,07(*)$ & $0,67(+)$ & 1998 \\
\hline 1999 & 1,03 & 1,21 & $-0,25$ & 0,35 & 1999 \\
\hline $2000(+)$ & 0,60 & $1,98(+)$ & $0,52(+)$ & 0,17 & $2000(+)$ \\
\hline 2001 & $-0,48(-)$ & $0,19(*)$ & $-0,65$ & 0,25 & 2001 \\
\hline 2002 & 0,43 & 1,62 & $-0,07\left(^{*}\right)$ & $-0,27$ & $2002(*)$ \\
\hline 2003(*) & $-0,15$ & $0,12(*)$ & $-0,48$ & $-0,16$ & 2003(-) \\
\hline 2004 & 0,38 & 0,72 & $-0,48$ & 0,32 & 2004 \\
\hline $2005(*)$ & $0,00(*)$ & $0,00(*)$ & $-0,33$ & 0,26 & 2005 \\
\hline 2006(-) & $-0,08(*)$ & $-0,73(-)$ & $-0,77(-)$ & 0,19 & 2006 \\
\hline $2007(+)$ & 1,20 & $1,96(+)$ & $-0,17$ & $0,52(+)$ & 2007 \\
\hline 2008 & $0,05(*)$ & 1,75 & $0,50(+)$ & $-0,09\left(^{*}\right)$ & 2008 \\
\hline 2009 & $1,28(+)$ & 0,61 & $-0,28$ & $-0,61(-)$ & 2009(-) \\
\hline 2010(-) & $-2,00(-)$ & $-1,99(-)$ & $-0,38$ & $0,05(*)$ & $2010(*)$ \\
\hline 2011(-) & $-0,67(-)$ & $-0,48(-)$ & $0,43(+)$ & 0,48 & 2011(+) \\
\hline $2012(+)$ & $1,50(+)$ & $1,84(+)$ & $-1,98(-)$ & $-0,54(-)$ & 2012(-) \\
\hline
\end{tabular}

AOI: Artic Oscillation Index; (+): Fase Positiva; (*): Fase Neutra; (-): Fase Negativa

$(1980,1981,1982,1985,2003,2005)$, en tanto que para la estación lluviosa las composiciones fueron: $\mathrm{AO}(+)(1983$, 1984, 1989, 1994, 2000, 2011), AO(-) (1993, 1995, 1997, 2003, 2009, 2012), y en la fase neutra $(1981,1986,1991$, 1992, 2002, 2010).

En el caso del AAOI basado en las estaciones de observación, se usó aquel que empleó 12 estaciones para calcular las medias zonales de la presión a nivel del mar en $40^{\circ} \mathrm{S}$ y $65^{\circ} \mathrm{S}$ (Marshall, 2003) (http://www.nerc-bas.ac.uk/ icd/gjma/sam.html), y para el AAOI basado en los reanálisis (NCEP/NCAR) (Kalnay, et al., 1996) se utilizó el índice definido como la diferencia mensual normalizada de la presión media zonal a nivel del mar entre $40^{\circ} \mathrm{S}$ y $70^{\circ} \mathrm{S}(\mathrm{Nan} \&$ Li, 2003) (tomado de: http://ljp.gcess.cn/dct/page/65609). En la Tabla 4 se presentan los índices estacionales calculados para el AAOI, que para la estación seca dan como 
resultado las siguientes composiciones: $\mathrm{AAO}(+)(1982$, $1999,2000,2008,2011,2012)$ y AAO(-) $(1980,1981,1983$, 1985, 1992, 2006), y para la fase neutra, el siguiente: (1984, $1990,1991,1997,2001,2004)$, en tanto que para la estación lluviosa las composiciones fueron: $\mathrm{AAO}(+)(1989$, 1993, 1998, 2008, 2010, 2012) у AAO(-) (1980, 1981,
$1988,1992,1994,2002)$, y para la fase neutra fue (1986, $1987,1991,1995,2000,2005)$. Los valores umbrales de las fases positiva y negativa de esta selección se presentan en la Tabla 2, y para los de la fase neutral se seleccionaron los años con los índices más próximos a cero, al igual que en los casos anteriores.

Tabla 4. Índices estacionales considerados para las composiciones de las fases de la Oscilación Antártica para el periodo de 1979 a 2012.

\begin{tabular}{|c|c|c|c|c|c|}
\hline & \multicolumn{4}{|c|}{ AAOI } & \\
\hline & \multicolumn{2}{|c|}{ Seca } & \multicolumn{2}{|c|}{ Lluviosa } & \\
\hline & Observación & Reanálisis & Observación & Reanálisis & \\
\hline 1980(-) & $-1,54(-)$ & $-1,70(-)$ & $-0,47$ & $-0,76(-)$ & $1980(-)$ \\
\hline 1981(-) & $-0,93(-)$ & $-0,84(-)$ & $-0,67(-)$ & $-0,96(-)$ & 1981(-) \\
\hline $1982(+)$ & $1,94(+)$ & $2,33(+)$ & 0,36 & $0,55(*)$ & 1982 \\
\hline 1983(-) & $-1,23(-)$ & $-0,48(-)$ & 0,46 & 1,41 & 1983 \\
\hline 1984(*) & $0,17(*)$ & 0,64 & $-0,15(*)$ & 0,80 & 1984 \\
\hline 1985(-) & $-0,74(-)$ & $-0,10\left(^{*}\right)$ & 0,57 & 1,47 & 1985 \\
\hline 1986 & $-0,36$ & 0,57 & $0,14(*)$ & $-0,26(-)$ & $1986(*)$ \\
\hline 1987 & $-0,75(-)$ & $0,11(*)$ & 0,24 & $0,32(*)$ & $1987(*)$ \\
\hline 1988 & 0,40 & 1,18 & $-2,53(-)$ & $-0,46(-)$ & 1988(-) \\
\hline 1989 & 0,83 & 1,59 & $0,99(+)$ & $2,31(+)$ & $1989(+)$ \\
\hline $1990(*)$ & $-0,03(*)$ & 0,61 & $-0,55$ & 0,62 & 1990 \\
\hline 1991(*) & $-0,07(*)$ & $0,49(*)$ & $-0,22$ & $0,04(*)$ & 1991(*) \\
\hline 1992(-) & $-0,73(-)$ & $-1,12(-)$ & $-0,79(-)$ & $-0,77(-)$ & 1992(-) \\
\hline 1993 & 0,40 & $-0,53(-)$ & $1,73(+)$ & $2,58(+)$ & $1993(+)$ \\
\hline 1994 & $1,16(+)$ & 1,43 & $-0,69(-)$ & $0,17(*)$ & 1994(-) \\
\hline 1995 & 0,38 & 1,13 & $-0,09(*)$ & 0,59 & $1995(*)$ \\
\hline 1996 & $0,32(*)$ & 1,53 & $-0,41$ & $0,22(*)$ & 1996 \\
\hline 1997(*) & 0,38 & $0,42(*)$ & 0,60 & 1,45 & 1997 \\
\hline 1998 & 0,33 & 1,36 & $1,28(+)$ & $2,73(+)$ & $1998(+)$ \\
\hline 1999(+) & $1,55(+)$ & $2,33(+)$ & $1,06(+)$ & 1,72 & 1999 \\
\hline $2000(+)$ & $2,10(+)$ & $2,64(+)$ & $-0,18(*)$ & 0,67 & $2000(*)$ \\
\hline 2001(*) & $-0,29(*)$ & $-0,04(*)$ & $0,18(*)$ & 2,07 & 2001 \\
\hline 2002 & 0,98 & 1,56 & $-1,60(-)$ & $-1,40(-)$ & 2002(-) \\
\hline 2003 & 0,33 & 0,58 & 0,20 & 1,33 & 2003 \\
\hline $2004(*)$ & $0,06(*)$ & $0,47(*)$ & 0,87 & 1,89 & 2004 \\
\hline 2005 & 0,63 & 0,92 & $-0,10(*)$ & 0,77 & $2005(*)$ \\
\hline 2006(-) & $-0,40$ & $-0,11(-)$ & 0,81 & 1,66 & 2006 \\
\hline 2007 & 0,46 & 0,77 & $-1,23(-)$ & $0,26(*)$ & 2007 \\
\hline $2008(+)$ & $1,05(+)$ & $1,72(+)$ & 0,97 & $2,33(+)$ & $2008(+)$ \\
\hline 2009 & 0,85 & 1,71 & $-0,25$ & 0,65 & 2009 \\
\hline 2010 & $-0,42$ & 0,73 & $2,02(+)$ & $4,15(+)$ & $2010(+)$ \\
\hline 2011(+) & $1,052(+)$ & $1,79(+)$ & $-0,23$ & 1,01 & 2011 \\
\hline 2012(+) & 1,04 & $1,81(+)$ & $1,23(+)$ & $2,37(+)$ & 2012(+) \\
\hline
\end{tabular}

AAOI: Antarctic Oscillation Index; (+): Fase Positiva; (*): Fase Neutra; (-): Fase Negativa 


\section{Resultados y discusión}

En la Tabla 5 se presenta la cuantificación semestral del aporte de humedad, $(|E-P<0|)$ de cada fuente oceánica a la precipitación en la región de análisis (Figura 1, mapa superior), de las cuales la que más humedad aporta es la MEXCAR en ambas estaciones (Durán-Quesada, et al., 2010). Sin embargo, cabe destacar que no solo la magnitud de esta contribución aumenta en la estación lluviosa (pues pasa de $17,2 \mathrm{~mm} /$ día en la época seca a $38,2 \mathrm{~mm} /$ día en la época lluviosa), sino también su porcentaje relativo comparado con el total de la estación que registra la precipitación proveniente de la MEXCAR. A esta le sigue la fuente NATL, que también aumenta la magnitud de su aporte de humedad en la estación lluviosa (pasando de 11,1 $\mathrm{mm} /$ día en la época seca a $17,7 \mathrm{~mm} /$ día en la época lluviosa), pero cuyo porcentaje relativo disminuye con respecto al total de la estación. Se destaca que estas son las dos principales fuentes oceánicas que aportan humedad a la región climática continental MAM, ya que el aporte de las fuentes NPAC y SPAC es muy pequeño. A pesar de ello, ambas fuentes incrementan no solo la magnitud de su aporte de humedad en la estación lluviosa (pasando de 1,0 y $0,5 \mathrm{~mm} /$ día en el periodo seco a 3,6 y $1,9 \mathrm{~mm} /$ día en el periodo lluvioso, respectivamente), sino también su porcentaje relativo de precipitación.

Los patrones climatológicos de la precipitación proveniente de estas fuentes oceánicas de humedad fueron estudiados por Gimeno, et al. $(2010,2013)$ desde una perspectiva global. Sin embargo, en el presente estudio se retomó el análisis con un enfoque regionalizado que consideró los periodos semestrales, cuyos resultados para el campo medio estacional de los sumideros de humedad se presentan en la Figura 1. En el caso de la MEXCAR durante la estación seca, se observa un aporte de humedad sobre todo el Caribe, con excepción de la península de Yucatán. Por el contrario, en la vertiente del Pacífico no hay transporte hacia la Sierra Madre Occidental ni hacia la del Sur, así como tampoco hasta El Salvador ni el Pacífico de Nicaragua. En la estación lluviosa la contribución de humedad proveniente de la MEXCAR se percibe en casi toda la región, donde existe un claro transporte hacia la Sierra Madre Occidental, exceptuando los territorios de Nayarit y Aguas Calientes, los cuales no se ven afectados por esta fuente. También cabe destacar que esta fuente no tiene influencia sobre Mérida, en la península de Yucatán, ni tampoco sobre las Antillas Mayores en ninguna de las estaciones, dado que el transporte de humedad hacia estos territorios proviene de la fuente NATL.

La cobertura espacial del aporte de humedad proveniente de la fuente NATL en la región es bastante semejante en ambas estaciones, con una contribución desde el norte hasta el sur y una fuerte influencia en la vertiente Caribe, lo que demuestra la gran importancia que tiene el aporte de humedad de esta fuente en América Central (DuránQuesada, et al., 2010). Castillo, et al. (2011, 2014b) analizaron los aspectos climatológicos del transporte de humedad desde esta fuente oceánica hacia la región continental y
Tabla 5. Cuantificación de la magnitud de los sumideros de humedad provenientes de las principales fuentes oceánicas que afectaron la región climática continental MAM en las estaciones seca (NDEFMA) y lluviosa (MJJASO) durante el periodo de 1979 a 2012

\begin{tabular}{lcccc}
\hline & \multicolumn{2}{c}{ mm/día } & \multicolumn{2}{c}{$\%$} \\
\cline { 2 - 5 } & Seca & Lluviosa & Seca & Lluviosa \\
\hline MEXCAR & 17,2 & 38,2 & 57,8 & 62,3 \\
NATL & 11,1 & 17,7 & 37,2 & 28,8 \\
NPAC & 1,0 & 3,6 & 3,3 & 5,8 \\
SPAC & 0,5 & 1,9 & 1,7 & 3,1 \\
& 29,7 & 61,4 & 100 & 100 \\
\hline
\end{tabular}

MEXCAR: Golfo de México - Mar Caribe; NPAC: Pacífico Norte; NATL: Atlántico Norte; SPAC: Pacífico Sur

evidenciaron que el movimiento estacional del anticiclón subtropical del Atlántico (North Atlantic Subtropical High, NASH) y la ITCZ son los elementos dinámicos reguladores del comportamiento de las precipitaciones generadas por el vapor de agua proveniente de esta fuente. En cuanto a las fuentes NPAC y SPAC, su pequeño aporte de humedad se da en lugares específicos de la región MAM, y cabe anotar que para la estación seca, la NPAC aporta humedad a Tucson y a Texas, en tanto que SPAC lo hace en Panamá y el Pacífico sur de Costa Rica. Por otra parte, en la estación lluviosa la NPAC influye en el Pacífico sur de México, en tanto que SPAC lo hace en la costa Pacífica desde el sur de México hasta Panamá.

La importancia de analizar los factores que gobiernan la variabilidad climática de esta región radica en que gran parte de esta zona geográfica se ha asociado con proyecciones de cambio climático como las reducciones medianas pero significativas, de 5 a $10 \%$, en la precipitación en el norte de América Central relacionada con el desplazamiento hacia el sur de la ITCZ en el periodo de 2050 a 2099 (Hidalgo, et al., 2013), así como con cambios significativos en la duración del MSD, el cual se prevé que aumentará más de una semana y su precipitación mínima disminuirá más del $26 \%$ en promedio en la mayoría de la zona del Pacífico de Nicaragua, Honduras, El Salvador y Guatemala (Maurer, $\boldsymbol{e t}$ al., 2017), lo que se traduce en condiciones más secas en los países del istmo centroamericano para finales del siglo XXI (Castillo, et al., 2018), incluido todo el territorio de Costa Rica (Castillo, et al., 2017).

En la Tabla 6 se muestran las correlaciones, con un valor umbral de \pm 0.29 , entre las series estacionales de tiempo de la magnitud de los sumideros de humedad en la región MAM y los índices de los modos de variabilidad climática con un nivel de confianza estadístico mayor al $95 \%$. Se puede observar que ENOS tuvo una influencia significativa en la estación seca en las fuentes NPAC y SPAC, en tanto que en la estación lluviosa su alcance se extendió hacia las cuatro fuentes. En el caso de los modos anulares, la AO 
afectó de forma significativa durante la estación seca en las fuentes MEXCAR y NATL, en tanto que la AAO tuvo un impacto mucho mayor en las fuentes NPAC y SPAC durante la estación seca que durante la lluviosa, pues resultó significativa solo con los índices obtenidos del reanálisis en esta época del año.

Con la finalidad de indagar si las correlaciones obtenidas entre los sumideros de humedad debidos a la NPAC y la SPAC con la AAO están asociadas con un elemento detonador en común (ENOS), se hizo una correlación parcial entre $(|E-P<0|)$ y AAO dejando constante el ONI. Además, se mantuvo el mismo valor umbral y el mismo intervalo de confianza estadística utilizados en los cálculos de la Tabla 6 , y se pudo observar (Tabla 7) que efectivamente se mantiene el mismo comportamiento, con excepción de la fuente NPAC que pierde su correlación en la estación lluviosa.

En la Tabla 8 se presenta la cuantificación de la modulación semestral de los modos de variabilidad climática en el transporte de humedad hacia la región MAM. Las representaciones esquemáticas de la influencia espacial de las fases positiva y negativa de las oscilaciones atmosféricas en la precipitación se calcularon a partir de las diferencias de las composiciones de estas fases con respecto a la composición de la fase neutral. Para dotar de significado estadístico a los resultados obtenidos, se aplicó una prueba de bootstrapping (Efron, 2003) con un nivel de confianza del intervalo mayor de $90 \%$. Dichos resultados se presentan en la Figura 3 para ENOS, en la Figura 4 para la AO y en la Figura 5 para la AAO. Cabe destacar que para la AO solo se muestran las fuentes MEXCAR y NATL, dado que NPAC y SPAC no mostraron diferencias significativas con respecto a la composición de los años neutros.

El Niño-Oscilación del Sur. Con respecto a los resultados que están dentro del nivel de confianza estadístico del $95 \%$ cabe resaltar la correlación positiva de ENOS con la precipitación proveniente de las fuentes NPAC y SPAC durante la estación seca, en la cual durante la fase de El Niño se registra un incremento en los sumideros de humedad, en tanto que La Niña ocurre una disminución. Este comportamiento de la humedad proveniente de la NPAC se puede establecer reconstruyendo los eventos extremos debidos a ENOS a partir de los anillos de los árboles de la Sierra Madre Occidental y el sur de las Grandes Llanuras, en donde los eventos cálidos (El Niño) favorecen las condiciones húmedas y frías desde octubre hasta marzo, mientras que en la fase fría (La Niña) el clima es opuesto (seco y cálido) (Stahle \& Cleaveland, 1993). Este comportamiento se explica por el efecto específico de ENOS en la interacción entre los sistemas atmosféricos de latitudes medias y los trópicos, lo que hace que el flujo del chorro subtropical que atraviesa el Golfo de México y el sureste de los Estados Unidos se intensifique durante los periodos de El Niño y se debilite en los de La Niña, y sugiere el aumento de la incursión de aire frío en la región durante los primeros y su disminución durante los segundos (Schultz, et al., 1998).
Tabla 6. Coeficientes de la correlación de Pearson entre las series estacionales de tiempo de los sumideros de humedad $(|\mathrm{E}-\mathrm{P}<0|)$ provenientes de las principales fuentes oceánicas que afectaron la región climática continental MAM y los índices de las oscilaciones atmosféricas de escala global durante el periodo de 1979 a 2012 con un nivel de confianza estadístico mayor al $95 \%$

\begin{tabular}{|c|c|c|c|}
\hline & \multicolumn{2}{|c|}{ ONI } & \\
\hline & Seca & Lluviosa & \\
\hline MEXCAR & ---- & $-0,39$ & \\
\hline NATL & ---- & 0,41 & \\
\hline NPAC & 0,65 & $-0,55$ & \\
\hline \multirow[t]{4}{*}{ SPAC } & 0,74 & $-0,37$ & \\
\hline & \multicolumn{2}{|c|}{ AOI } & \\
\hline & Observación & Reanálisis & \\
\hline & \multicolumn{2}{|c|}{ Seca } & \\
\hline MEXCAR & 0,40 & 0,51 & \\
\hline \multirow[t]{4}{*}{ NATL } & 0,36 & 0,46 & \\
\hline & & AAOI & \\
\hline & Observación & Reanálisis & Reanálisis \\
\hline & \multicolumn{2}{|c|}{ Seca } & Lluviosa \\
\hline NPAC & $-0,58$ & $-0,55$ & 0,38 \\
\hline SPAC & $-0,73$ & $-0,69$ & 0,47 \\
\hline
\end{tabular}

MEXCAR: Golfo de México - Mar Caribe; NPAC: Pacífico Norte; NATL: Atlántico Norte; SPAC: Pacífico Sur; ONI: Oceanic Niño Index; AOI: Artic Oscillation Index; AAOI: Antarctic Oscillation Index

Tabla 7. Coeficientes de la correlación parcial de Pearson entre las series estacionales de tiempo de los sumideros de humedad $(|\mathrm{E}-\mathrm{P}<0|)$ provenientes de las principales fuentes oceánicas que afectaron la región climática continental MAM y la AAOI al mantener constante el índice ONI durante el periodo de 1979 a 2012 con un nivel de confianza estadístico mayor al $95 \%$

\begin{tabular}{|c|c|c|c|}
\hline & \multicolumn{3}{|c|}{ AAOI/ONI } \\
\hline & Observación & Reanálisis & Reanálisis \\
\hline & \multicolumn{2}{|c|}{ Seca } & Lluviosa \\
\hline NPAC & $-0,40$ & $-0,38$ & -- \\
\hline SPAC & $-0,63$ & $-0,59$ & 0,41 \\
\hline
\end{tabular}

NPAC: Pacífico Norte; SPAC: Pacífico Sur; ONI: Oceanic Niño Index; AAOI: Antarctic Oscillation Index

Dado que el CLLJ y el chorro de bajo nivel del Chocó (Chocó low level jet, CJ) (Poveda \& Mesa, 1999) son los principales transportadores de humedad hacia la región de América Central, el estudio de sus mecanismos es clave para determinar la estacionalidad de la lluvia forzada a gran escala sobre esta región (Durán-Quesada, et al., 2017b). En el caso del comportamiento del transporte de humedad proveniente de la SPAC durante la estación seca en las fases de ENOS, podría decirse que responde a la dinámica de los vientos en niveles bajos debida al CLLJ y al CJ, con una reducción de la magnitud de los vientos del CLLJ durante 
Tabla 8. Cuantificación de la modulación estacional de las oscilaciones atmosféricas de escala global en los sumideros de humedad $(|\mathrm{E}-\mathrm{P}<0|)$ provenientes de las principales fuentes oceánicas con impacto en la región climática continental MAM durante el periodo de 1979 a 2012

\begin{tabular}{|c|c|c|c|c|c|c|c|c|}
\hline & \multicolumn{2}{|c|}{ mm/día } & \multicolumn{2}{|c|}{$\%$} & \multicolumn{2}{|c|}{$\mathrm{mm} /$ día } & \multicolumn{2}{|c|}{$\%$} \\
\hline & Seca & Lluviosa & Seca & Lluviosa & Seca & Lluviosa & Seca & Lluviosa \\
\hline & \multicolumn{4}{|c|}{ Niño } & \multicolumn{4}{|c|}{ Niña } \\
\hline MEXCAR & 18,1 & 36,1 & 52,5 & 61,0 & 18,0 & 46,2 & 62,2 & 64,4 \\
\hline NATL & 12,1 & 19,5 & 35,0 & 33,0 & 10,3 & 15,5 & 35,5 & 21,6 \\
\hline NPAC & 3,4 & 2,0 & 9,9 & 3,4 & 0,4 & 7,0 & 1,5 & 9,8 \\
\hline \multirow[t]{3}{*}{ SPAC } & 0,9 & 1,5 & 2,6 & 2,6 & 0,2 & 3,1 & 0,8 & 4,3 \\
\hline & 34,5 & 59,2 & 100,0 & 100,0 & 28,9 & 71,8 & 100,0 & 100,0 \\
\hline & \multicolumn{4}{|c|}{$\mathbf{A O}(+)$} & \multicolumn{4}{|c|}{ AO(-) } \\
\hline MEXCAR & 22,6 & 43,5 & 61,9 & 63,7 & 14,3 & 37,0 & 59,9 & 62,1 \\
\hline NATL & 12,8 & 19,7 & 35,0 & 28,8 & 8,9 & 17,6 & 37,2 & 29,5 \\
\hline NPAC & 0,7 & 3,5 & 2,0 & 5,2 & 0,4 & 2,9 & 1,7 & 4,9 \\
\hline \multirow[t]{3}{*}{ SPAC } & 0,4 & 1,6 & 1,1 & 2,4 & 0,3 & 2,1 & 1,2 & 3,5 \\
\hline & 36,6 & 68,3 & 100,0 & 100,0 & 23,9 & 59,6 & 100,0 & 100,0 \\
\hline & \multicolumn{4}{|c|}{$\mathbf{A A O}(+)$} & \multicolumn{4}{|c|}{ AAO(-) } \\
\hline MEXCAR & 16,7 & 39,6 & 62,3 & 59,7 & 19,4 & 36,0 & 55,0 & 62,0 \\
\hline NATL & 9,7 & 17,3 & 36,1 & 26,1 & 12,5 & 17,6 & 35,5 & 30,3 \\
\hline NPAC & 0,3 & 6,4 & 1,1 & 9,7 & 2,5 & 3,0 & 7,1 & 5,2 \\
\hline \multirow[t]{2}{*}{ SPAC } & 0,1 & 3,0 & 0,5 & 4,5 & 0,8 & 1,4 & 2,4 & 2,5 \\
\hline & 26,8 & 66,3 & 100,0 & 100,0 & 35,3 & 58,0 & 100,0 & 100,0 \\
\hline
\end{tabular}

MEXCAR: Golfo de México - Mar Caribe; NATL: Atlántico Norte; NPAC: Pacífico Norte; SPAC: Pacífico Sur; AO: Artic Oscillation; AAO: Antarctic Oscillation

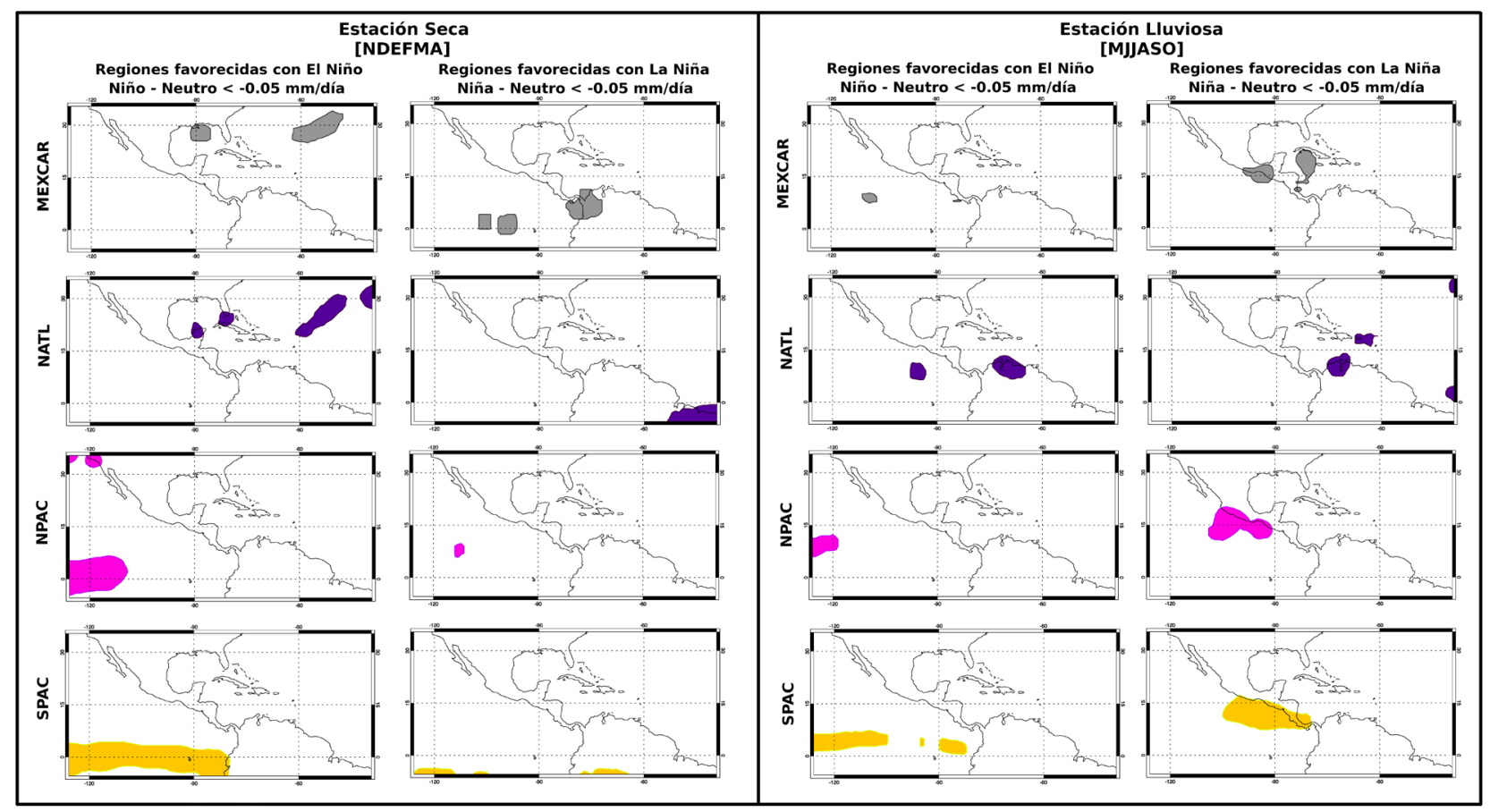

Figura 3. Diferencias entre las composiciones de las fases positiva y negativa con respecto a la composición de la fase neutral para El NiñoOscilación del Sur. Solo se graficaron los valores por debajo de $-0,05 \mathrm{~mm} /$ día y que resultaron dentro del nivel de confianza estadístico por encima del $90 \%$ en la prueba de bootstrapping al permutar la serie de tiempo 1.000 veces 


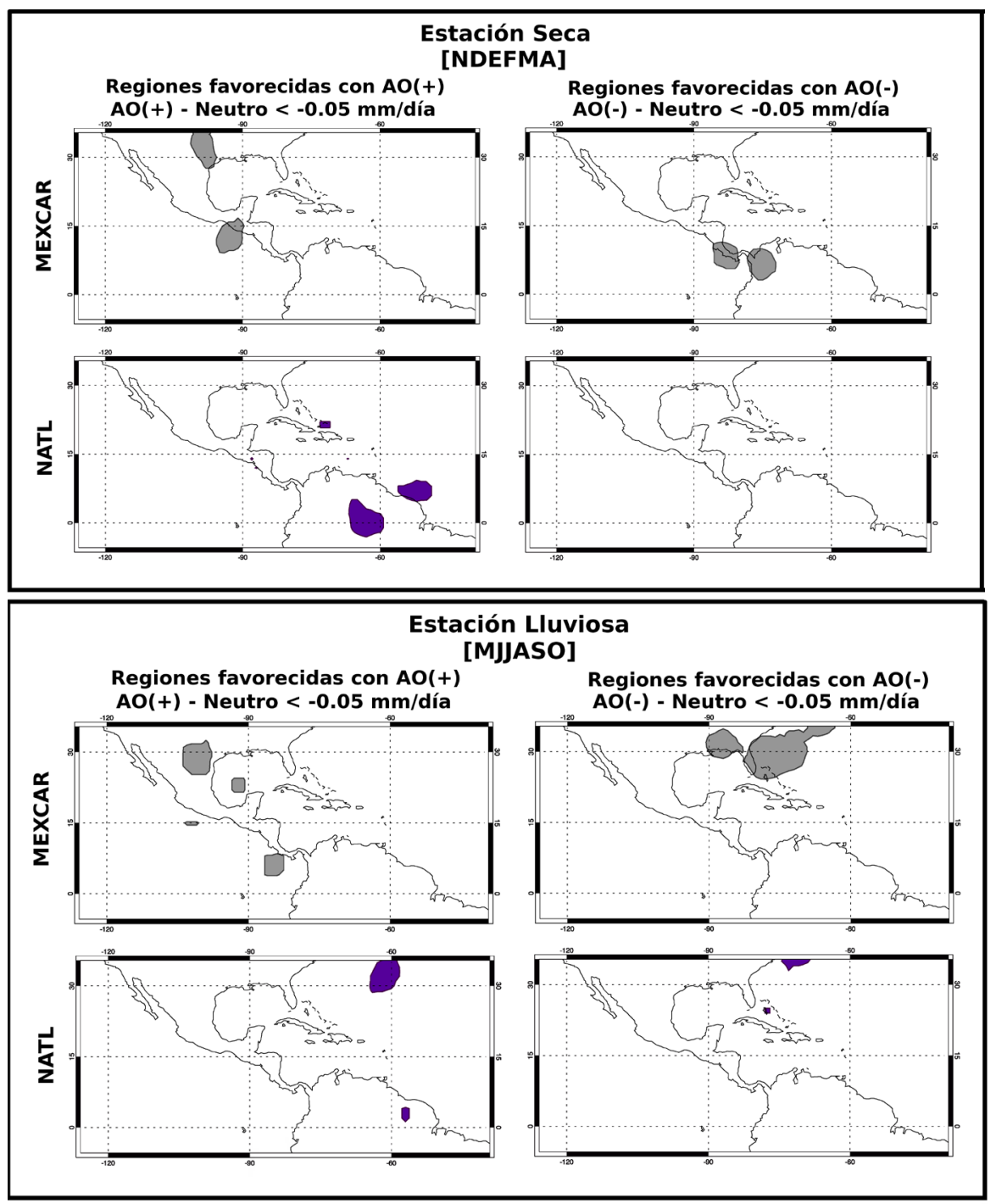

Figura 4. Diferencias entre las composiciones de las fases positiva y negativa con respecto a la composición de la fase neutral para la Oscilación Ártica. Solo se graficaron los valores menores a $-0,05 \mathrm{~mm} /$ día que quedaban dentro del nivel de confianza estadístico por encima del $90 \%$ en la prueba de bootstrapping al permutar la serie de tiempo 1.000 veces

El Niño y un incremento durante La Niña (Amador, et al., 2003), lo cual produce un movimiento ascendente sobre el lado del Pacífico que aumenta la convección durante el primero, y uno descendente que la disminuye durante La Niña (Sáenz \& Durán-Quesada, 2015), pues se ve favorecida por el aumento (y la disminución en el caso de La Niña) del transporte de humedad desde la SPAC hacia el Pacífico del sur de América Central a través del CJ.

En su momento, Hales (1972) y Brenner (1974) sugirieron que la lluvia del verano boreal extendido en Arizona se debía a la humedad que se origina en el océano Pacífico tropical oriental y es transportada en los niveles bajos a través del golfo de California. Más tarde, Douglas, et al. (1993) y Bowen (1996) propusieron que parte del transporte de humedad y la lluvia que se extiende hacia el norte desde el noroeste de México hasta el suroeste de los Estados Unidos podía estar asociada con la circulación monzónica. Hoy esto se ha corroborado al constatar la influencia de los aumentos del contenido de humedad en la baja tropósfera de la piscina de agua cálida del hemisferio occidental, lo que alimenta el monzón de América del Norte (Wang \& Enfield, 2001, 2003; Durán-Quesada, et al., 2017a).

La correlación negativa entre el transporte de humedad desde la fuente NPAC y ENOS durante la estación lluviosa fue analizada por Gutzler \& Preston (1997) a partir de las anomalías del manto de nieve durante el invierno y la primavera boreales, con lo que se evidenció cómo la fase madura de El Niño inhibe la siguiente circulación monzónica del verano boreal sobre Nuevo México. Por el contrario, un déficit de nieve asociado con La Niña promovería una circulación monzónica anómalamente fuerte, resultados que 


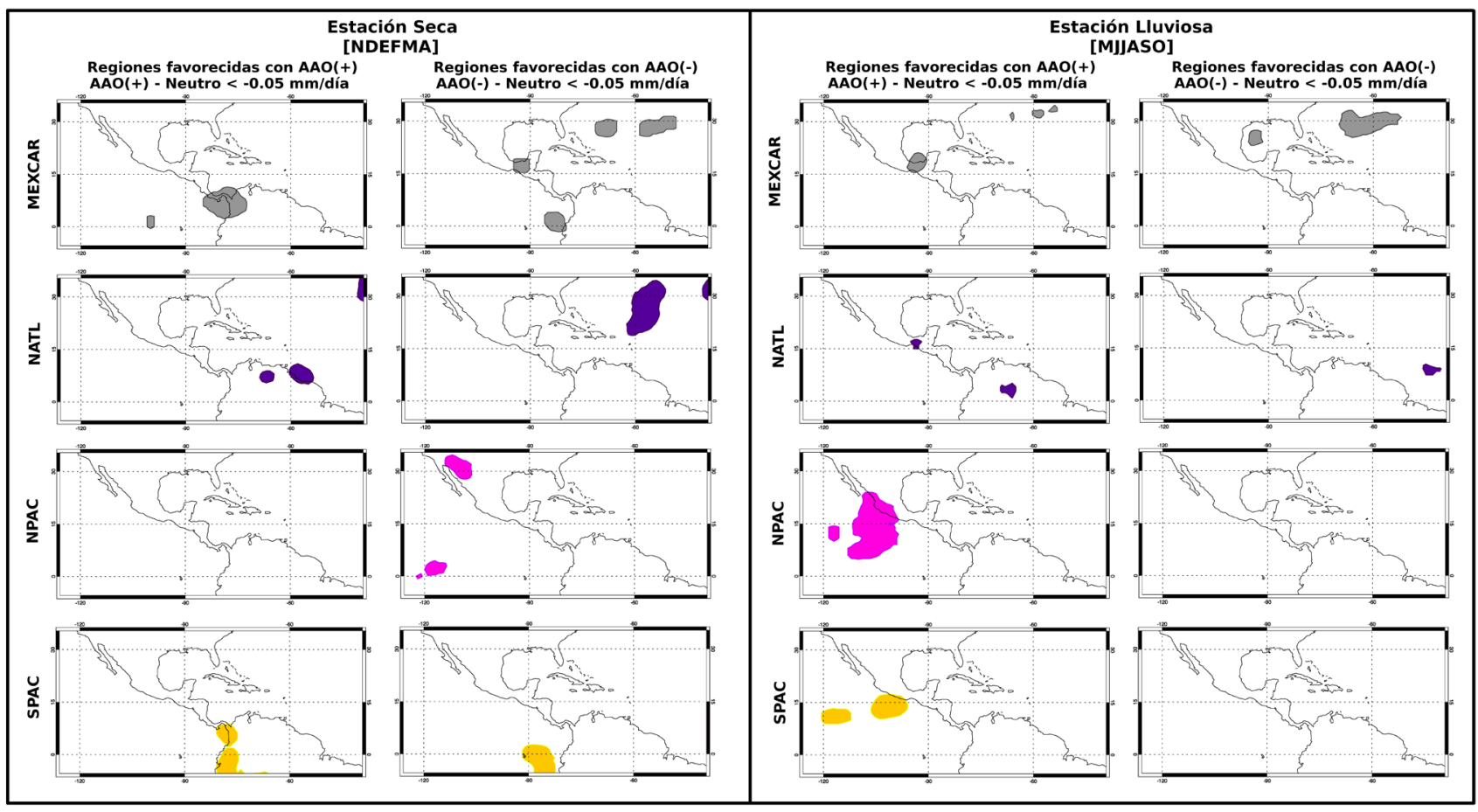

Figura 5. Diferencias entre las composiciones de las fases positiva y negativa con respecto a la composición de la fase neutral para la Oscilación Antártica. Solo se graficaron los valores menores a $-0,05 \mathrm{~mm} /$ día que quedaban dentro del nivel de confianza estadístico por encima del $90 \%$ en la prueba de bootstrapping al permutar la serie de tiempo 1.000 veces

luego fueron confirmados por Gochis, et al. (2007), revelando como ENOS ejerce una influencia modesta pero estadísticamente significativa en la corriente de flujo del monzón de América del Norte.

La fuente NATL se correlaciona positivamente con ENOS en el transporte de humedad en el periodo lluvioso, mientras que la SPAC lo hace de manera negativa. Esto se debe al comportamiento de las variaciones en la intensidad del CLLJ que tiene una correlación positiva con esta oscilación en el verano boreal (Amador, et al., 2003), y a la correlación negativa de la intensidad del CJ en el otoño boreal (Poveda \& Mesa, 1999); ello produce un movimiento descendente durante El Niño y ascendente durante La Niña sobre el lado del Pacífico, con lo que se disminuye la convección profunda durante el primero y la aumenta durante el segundo fenómeno (Sáenz \& Durán-Quesada, 2015). Esta dinámica es totalmente congruente con los resultados que evidencian cómo durante la fase de El Niño se registra un superávit (o un déficit durante La Niña) en el transporte de humedad hacia esta región que lleva a que la humedad proveniente de la fuente NATL alcance regiones del océano Pacífico y los aportes de la fuente SPAC se mantengan en las latitudes ecuatoriales, en tanto que en la fase de La Niña ocurre lo contrario, pues no se transporta tanta humedad desde la fuente NATL y la fuente SPAC afecta la vertiente del Pacífico desde el sur de México hasta Panamá.

La correlación negativa de la magnitud de los sumideros de humedad provenientes de la fuente MEXCAR durante ENOS se debe a la cobertura de esta fuente sobre el territorio mexicano en el curso de la estación lluviosa, lo cual se explica por los diversos mecanismos asociados con El Niño que resultan en anomalías negativas de precipitación sobre la mayor parte de México. Entre tales mecanismos cabe mencionar una subsidencia reforzada por un desplazamiento hacia el sur de la ITCZ, vientos alisios más intensos de lo normal, un menor número de ciclones tropicales en el mar intra-americano (Intra-Americas Sea, IAS) y una reducción en la humedad relativa, lo que puede resultar en graves sequías. Por otro lado, durante las fases de La Niña, las condiciones del clima en México regresan a la normalidad e, incluso, pueden resultar en una precipitación por encima del promedio (Magaña, et al., 2003).

Oscilación Ártica. El fortalecimiento o debilitamiento de la $\mathrm{AO}$ es el fenómeno que más impacta la variabilidad climática durante el invierno boreal, especialmente en el noreste de América, el Atlántico y Eurasia (Van Loon \& Rogers, 1978; Wallace \& Gutzler, 1981), produciendo variaciones en los fenómenos atmosféricos a escalas interanuales y más largas (Thompson \& Wallace, 2001). Dicho impacto se ha evidenciado en diversos estudios sobre el clima regional y hemisférico en esta época del año, entre los cuales se destacan los de Hurrell (1995, 1997), Kodera, et al. (1999), Rodwell, et al. (1999) y Xie, et al. (1999).

Las características de la circulación de invierno (verano) en las latitudes medias del hemisferio norte son más fuertes (débiles), más baroclínicas (barotrópicas) y los chorros 
están menos (más) zonalmente orientados, lo que inhibe (promueve) la formación de trenes de ondas globales. Estas configuraciones están más (menos) influenciadas por la variabilidad de la SST tropical, y es menos (más) sensible a la retroalimentación por la interacción entre la tierra y la atmósfera que involucra la humedad del suelo o la cobertura de nieve (Coumou, et al., 2018).

El agente impulsor climático más dominante en América Central es la NASH (Taylor \& Alfaro, 2005; Amador, et al., 2006; 2016), la cual se correlaciona directamente con la AO. Su influencia puede extenderse a los IAS que rodean la región mesoamericana, como se aprecia en la Figura 1. Este comportamiento se debe al fortalecimiento o debilitamiento de los vientos alisios que se encuentran cerca de flanco ecuatorial y modulan el clima de esta región (Alfaro, et al., 2018).

La correlación positiva entre el transporte de humedad desde la MEXCAR y la NATL y el AOI en la estación seca (Tabla 6) se debe a las características regionales que hacen que durante el invierno boreal el balance del flujo de humedad sobre los IAS sea principalmente zonal, con la humedad proveniente del Atlántico Norte tropical que se evapora localmente sobre ellos y logra un equilibrio en el transporte hacia el Pacífico sobre México y América Central (Mestas-Núñez, et al., 2007) que puede verse afectado por la fase $\mathrm{AO}(+)(\mathrm{AO}(-))$, la cual provoca un aumento (disminución) en el transporte de humedad hacia la región.

Además, según Zárate-Hernández (2003), este comportamiento guarda relación tanto con el número de entradas de los frentes fríos como con las profundidades latitudinales que alcanzan estos sistemas sobre la región MAM. Así, la fase $\mathrm{AO}(-)$ promueve anomalías negativas de la presión en la superficie sobre el Atlántico y el mar Caribe que debilitan el flujo de los alisios en la troposfera baja de esas áreas, generando anomalías de viento del oeste que permiten la penetración de un mayor número de empujes fríos durante el invierno boreal.

Por otro lado, durante el verano boreal se reduce el gradiente de temperatura entre la zona polar y la ecuatorial, lo que hace que se estrechen y se debiliten los vientos del oeste en los niveles altos, y provoca que el vórtice polar estratosférico esté prácticamente ausente (Coumou, et al., 2018). Por ello se considera que la estratosfera no influye en el clima boreal de verano (Kidston, et al., 2015) y es la posición anómala de la SST tropical la que determina dónde tiene lugar la convección profunda más fuerte asociada con los cambios en la circulación de Walker (Coumou, et al., 2018).

Todos estos elementos explican por qué no se encontró una correlación con el AOI durante la estación lluviosa. Sin embargo, sabiendo que la configuración de la $\mathrm{AO}$ es la misma en el invierno que en el verano boreal, pero debilitada y con una climatología dinámica opuesta (Coumou, et al., 2018), hace que nos permite explicar cómo el patrón espacial encontrado (Figura 4) responde muy ajustadamente al aumento (disminución) de los vientos alisios en la fase $\mathrm{AO}(+)(\mathrm{AO}(-))$, lo que promueve un fortalecimiento (debilitamiento) en el transporte de la humedad desde la MEXCAR hacia el Pacífico. Por último, en la fase AO(-) se da un incremento del transporte de humedad desde la MEXCAR y la NATL hacia las regiones extratropicales a través de las trayectorias de las tormentas que se ven intensificadas con la reducción de los vientos alisios.

Oscilación Antártica. La AAO es el principal modo de variabilidad de la circulación extratropical del hemisferio sur y se caracteriza por una estructura profunda, zonalmente simétrica o anular, con variaciones en la altura geopotencial de signo opuesto entre la región del casquete polar y el anillo zonal circundante centrado cerca de los $45^{\circ}$ de latitud (Thompson \& Wallace, 2000).

Aunque la AO y la AAO son estructuras notablemente similares, tanto en la altura geopotencial zonal media y los campos de viento zonales como en las circulaciones meridionales medias, y están presentes durante todo el año en la troposfera, difieren en sus periodos de amplificación (atenuación) de la altura en dirección a la estratosfera, de diciembre a febrero (DEF) para la $\mathrm{AO}$ y en noviembre $(\mathrm{N})$ para la $\mathrm{AAO}$ (de junio a agosto, JJA, para la $\mathrm{AO}$ y en febrero y marzo, FM, para la AAO), que conducen a una fuerte interacción entre el flujo medio y las ondas planetarias. Durante estos periodos de activación (inactivación) dichas configuraciones anulares modulan la fuerza de la circulación lagrangiana media en la estratosfera inferior, la altura de la tropopausa sobre latitudes medias y altas, la columna total de ozono y la fuerza de los vientos alisios de sus respectivos hemisferios (Thompson \& Wallace, 2000).

El comportamiento climatológico estacional de los vientos a $925 \mathrm{hPa}$ sobre los IAS puede consultarse en DuránQuesada, et al. (2017b), quienes evidencian claramente las variaciones del CLLJ, el CJ, los vientos alisios del sur y el posicionamiento de la ITCZ en el océano Pacífico tropical oriental. El periodo de máxima intensificación del CLLJ es en DEF, mientras que el del CJ se da en SON. Durante DEFMAM la ITCZ se posiciona por encima de los $5^{\circ} \mathrm{N}$, en tanto que en JJASON se ubica por encima de $\operatorname{los} 8^{\circ} \mathrm{N}$ $\mathrm{y}$, además, los vientos alisios del sur se observan curvados hacia el este después de cruzar el ecuador (configuración que promueve el transporte de humedad desde el Pacífico hacia regiones continentales del hemisferio norte cercanas al flanco ecuatorial). Asimismo, Marsh, et al. (2018) presentan la climatología estacional de los vientos a 925 hPa sobre América del Sur y anotan cómo los vientos alisios en DEF tienen una componente meridional más marcada, bordean la costa del Pacífico e impactan directamente el golfo de Arica, en tanto que en JJA este componente meridional se debilita, lo que hace que los vientos sean prácticamente de dirección sureste.

El impacto regional que tiene la $\mathrm{AAO}$ en el hemisferio sur se ha documentado ampliamente (Gillett, et al., 2006), pero no así las posibles teleconexiones que puedan existir entre esta oscilación y las regiones del hemisferio norte. Algunos trabajos pioneros, como el de 
Nan \& Li (2003) y Wu, et al. (2009), han evidenciado la existencia de correlaciones significativas entre el AAOI y las precipitaciones en China: los primeros encontraron una correlación positiva entre el AAOI de la primavera boreal y las precipitaciones del verano boreal en el valle del río Yangtze, en tanto que los segundos revelaron una correlación negativa entre el AAOI del otoño boreal y la intensidad de su monzón de invierno. Sin embargo, las correlaciones entre el transporte de humedad hacia la región MAM y el AAOI no se habían determinado anteriormente.

La correlación negativa encontrada entre el transporte de humedad desde la NPAC y la SPAC y el AAOI en la estación seca (Tabla 6), se debe a que en la fase $\mathrm{AAO}(+)$ (AAO(-)) aumenta (disminuye) el anticiclón subtropical del Pacífico Sur que intensifica (debilita) vientos alisios del sur encargados de transportar la humedad desde el Pacífico Sur hacia la ITCZ. Sabiendo que en esta época del año los vientos alisios tienen un gran componente meridional que bordea la costa del Pacífico, su aumento (disminución) provoca que ingrese mayor (menor) humedad a través del golfo de Arica y se transporte hacia el norte sobre la cordillera de los Andes, con un impacto directo sobre los países costeros (Figura 5), que se refleja en una disminución (aumento) del transporte de humedad hacia la región MAM debido al posicionamiento anómalo de la ITCZ y el anticiclón subtropical del Pacífico Norte, los cuales se ven desplazados hacia el sur (norte).

Por otra parte, en la estación lluviosa la correlación positiva entre el transporte de humedad desde la NPAC y la SPAC y el AAOI (Tabla 6) se debe, asimismo, a la intensificación (debilitamiento) de los vientos alisios del sur en la fase $\mathrm{AAO}(+)(\mathrm{AAO}(-))$, dado que para este periodo ellos son los encargados de transportar la humedad desde el Pacífico hacia la región MAM, favorecidos por las condiciones climatológicas del CLLJ, el CJ y su configuración de curvatura hacia el este después de cruzar el ecuador, lo cual fortalece el transporte de humedad hacia el noreste.

Dichos resultados son independientes de la señal del ONI, dado que durante la estación seca la correlación negativa entre el AAOI y el transporte de humedad desde las fuentes NPAC y SPAC se mantuvo a pesar del posible forzamiento que pueda tener ENOS como modo dominante de la variabilidad climática regional (Tabla 7), en tanto que en la estación lluviosa la correlación positiva resultó ser más débil, pues solo fue significativa para los índices del reanálisis (Tabla 6) y registró una pérdida de la señal en la correlación parcial para la fuente NPAC (Tabla 7).

Esta investigación ha evidenciado cómo la AAO también tiene un papel importante en las variaciones interanuales y ha permitido vislumbrar la influencia de esta oscilación en la precipitación que llega a la región MAM principalmente a través de la fuente SPAC. Inclusive si se considera el posible forzamiento de ENOS como modo dominante de la variabilidad climática regional y el planteamiento de Fogt \& Bromwich (2006) en el sentido de que esta teleconexión no es más que un acople entre la variabilidad decadal de
ENOS y la latitud alta del Pacífico Sur que gobierna la AAO. Este novedoso resultado es un aliciente para futuros trabajos que puedan mejorar el entendimiento del impacto de la AAO sobre la región MAM.

\section{Conclusiones}

En este estudio se utilizó el modelo lagrangiano FLEXPART alimentado con datos del reanálisis ERA-Interim para analizar la influencia semestral que tiene la variabilidad climática en el transporte de humedad desde las principales fuentes oceánicas que afectan la región continental MAM. Se encontró que la fuente que más humedad aporta a la región es la MEXCAR en ambas estaciones, seguida de la fuente NATL y, con aportes relativamente pequeños en comparación con las anteriores, las fuentes NPAC y SPAC.

Se pudo determinar que las cuatro fuentes incrementan la magnitud de su aporte de humedad durante la estación lluviosa. Sin embargo, a pesar de que en el periodo lluvioso las fuentes MEXCAR, NPAC y SPAC también aumentaron su porcentaje relativo con respecto al total de la estación que representa la precipitación proveniente de cada una de ellas, el porcentaje proveniente de la fuente NATL disminuyó en esa época del año.

Además, se estudiaron los patrones de teleconexión semestral con impacto en Mesoamérica debido a las oscilaciones atmosféricas de escala global e interanual, y se evidenció que ENOS es el modo dominante que afecta el suministro de humedad para la precipitación en la región a través de la modulación de los fenómenos regionales; dicho resultado es coherente con lo obtenido por Magaña, et al. (2003) para México y Durán-Quesada, et al. (2017b) para América Central.

Se encontró que ENOS influye más durante el periodo lluvioso que durante el seco, dado que en este se obtuvo una correlación positiva con el transporte de humedad a partir únicamente de dos fuentes (NPAC y SPAC). En el periodo lluvioso, por el contrario, se registró una correlación con las cuatro fuentes: negativa con MEXCAR, NPAC y SPAC y positiva con NATL, resultados estos que son muy acordes con la dinámica atmosférica regional.

En el caso de la AO se vislumbró una correlación positiva con las fuentes MEXCAR y NATL para la época seca, la cual está directamente relacionada con el movimiento latitudinal de la NASH, el cual influye en la intensidad de los vientos alisios. Esta correlación ya había sido estudiada a través de la NAO por Mestas-Núñez, et al. (2007), aunque con un resultado estadísticamente débil.

Por último, el resultado más relevante, que no se había reportado antes para la región MAM, es la correlación negativa de la AAO durante la estación seca con el transporte de humedad desde las fuentes NPAC y SPAC, la cual prevalece a pesar del posible forzamiento de ENOS como modo dominante de la variabilidad climática regional. En el periodo lluvioso la correlación es positiva y más débil, dado que fue significativa solo para los índices del reanálisis y con 
una pérdida de señal en la correlación parcial para la fuente NPAC. Al igual que con la AO, estas correlaciones están directamente relacionadas con el movimiento latitudinal del anticiclón subtropical del Pacífico Sur, el cual influye en la intensidad de los vientos alisios. Esta teleconexión tiene utilidad potencial para futuros estudios como predictor del comportamiento de la distribución de la precipitación, principalmente durante su periodo inicial y su primer máximo de precipitación, dada la alta correlación encontrada para este periodo.

\section{Contribución de los autores}

Rodrigo Castillo procesó la información y realizó los análisis. Raquel Nieto y Luis Gimeno diseñaron y dirigieron la investigación y Anita Drumond realizó las simulaciones.

\section{Conflicto de intereses}

Los autores manifiestan no tener conflicto de intereses con relación de este trabajo.

\section{Agradecimientos}

Los autores desean expresar su reconocimiento por el apoyo de los proyectos VI-B6147, VI-B7605, VI-B9454 y VI-B9609, de la Universidad de Costa Rica, los cuales permitieron al autor principal la elaboración del manuscrito. Agradecen, asimismo, a la Xunta de Galicia por financiar parcialmente esta investigación a través del proyecto CHEGA, y al Gobierno español que lo hizo a través del proyecto TRAMO, ambos cofinanciados por FEDER.

\section{Referencias}

Alfaro, E. J., Chourio, X., Muñoz, Á. G., Mason, S. J. (2018). Improved seasonal prediction skill of rainfall for the Primera season in Central America. International Journal of Climatology. 38: e255-e268. Doi: 10.1002/joc.5366

Amador, J. A. (2008). The Intra-Americas Sea low-level jet. Annals of the New York Academy of Sciences. 1146 (1): 153-188. Doi: 10.1196/annals. 1446.012

Amador, J. A., Alfaro, E. J., Lizano, O. G., Magaña, V. O. (2006). Atmospheric forcing of the eastern tropical pacific: A review. Progress in Oceanography. 69 (2): 101-142. Doi: 10.1016/j.pocean.2006.03.007

Amador, J. A., Chacón, R. E., Laporte, S. (2003). Climate and climate variability in the Arenal River basin of Costa Rica. In Climate and Water (pp. 317-349). Springer, Dordrecht.

Amador, J. A., Durán-Quesada, A., Rivera, E., Mora, G., Sáenz, F., Calderón, B., Mora, N. (2016). The easternmost tropical Pacific. Part ii: Seasonal and intraseasonal modes of atmospheric variability. Rev. Biol. Trop. 64 (Supplement 1): S23-S57.

Barker, T., Bashmakov, I., Bernstein, L., Bogner, J., Bosch, P., Dave, R., Davidson, O., Fisher, B., Grubb, M., Gupta, S., et al. (2007). Summary for policymakers. In Climate Change 2007: Mitigation of Climate Change: Contribution of Working Group III to the Fourth Assessment Report of the Intergovernmental Panel on Climate Change. Cambridge University Press.
Bengtsson, L., Hagemann, S., Hodges, K. I. (2004). Can climate trends be calculated from reanalysis data? Journal of Geophysical Research: Atmospheres. 109: (D11). Doi: 10.1029/2004JD004536

Bowen, B. M. (1996). Rainfall and climate variation over a sloping New Mexico plateau during the North American monsoon. Journal of Climate. 9 (12): 3432-3442. Doi: 10.1175/1520-0442(1996)009<3432:RACVOA $>2.0 . C O ; 2$

Brenner, I. S. (1974). A surge of maritime tropical airgulf of California to the southwestern United States. Monthly Weather Review. 102 (5): 375-389. Doi: 10.1175/1520-0493(1974)102<0375:ASOMTA >2.0.CO;2

Castillo, R., Amador, J., Durán-Quesada, A. (2017). Costa Rica rainfall in future climate change scenarios. In AGU Fall Meeting Abstracts.

Castillo, R., Montero, R., Amador, J., Durán, A. M. (2018). Cambios futuros de precipitación y temperatura sobre América Central y el Caribe utilizando proyecciones climáticas de reducción de escala estadística. Revista de Climatología. 18: 1-12.

Castillo, R., Nieto, R., Drumond, A. (2011). Análisis lagrangiano del comportamiento de los sumideros de humedad debidos a la fuente del Atlántico Norte para las estaciones de invierno y verano durante el periodo 1980-2000. Avances en ciencias de la tierra. 2: 39-51.

Castillo, R., Nieto, R., Drumond, A., Gimeno, L. (2014b). Estimating the temporal domain when the discount of the net evaporation term affects the resulting net precipitation pattern in the moisture budget using a 3-d Lagrangian approach. PloS One. 9 (6): e99046. Doi: 10.1371/journal. pone.0099046

Castillo, R., Nieto, R., Drumond, A., Gimeno, L. (2014a). The role of the ENSO cycle in the modulation of moisture transport from major oceanic moisture sources. Water Resources Research. 50 (2): 1046-1058. Doi: 10.1002/2013WR013900

Coen, E. (1973). El floklore costarricense relativo al clima. Revista de la Universidad de Costa Rica. 35: 135-145.

Coumou, D., Di Capua, G., Vavrus, S., Wang, L., Wang, S. (2018). The influence of Arctic amplification on midlatitude summer circulation. Nature Communications. 9 (1): 1-12. Doi: 10.1038/s41467-018-05256-8

Dee, D. P., Uppala, S. M., Simmons, A., Berrisford, P., Poli, P., Kobayashi, S., Andrae, U., Balmaseda, M., Balsamo, G., Bauer, d. P., et al. (2011). The era-interim reanalysis: Configuration and performance of the data assimilation system. Quarterly Journal of the Royal Meteorological Society. 137 (656): 553-597. Doi: 10.1002/qj.828

Durán-Quesada, A. M., Castillo, R., Hundsdoerfer, M., Gimeno, L. (2017a). CLLJ and WHWP heat content as a constraint to North American monsoon activation moisture supply. First International Electronic Conference on the Hydrological Cycle, 4856. Doi: 10.3390/CHyCle-2017-04856

Durán-Quesada, A. M., Gimeno, L., Amador, J. (2017b). Role of moisture transport for Central American precipitation. Earth System Dynamics. 8 (1): 147-161. Doi: 10.5194/esd8-147-2017

Durán-Quesada, A. M., Gimeno, L., Amador, J., Nieto, R. (2010). Moisture sources for Central America: Identification of moisture sources using a Lagrangian analysis technique. Journal of Geophysical Research: Atmospheres. 115 (D5). Doi: 10.1029/2009JD012455 
Efron, B. (2003). Second thoughts on the bootstrap. Statistical Science. 18 (2): 135-140. Doi: 10.1214/ss/1063994968

Fogt, R. L. \& Bromwich, D. H. (2006). Decadal variability of the ENSO teleconnection to the high-latitude south pacific governed by coupling with the Southern Annular Mode. Journal of Climate. 19 (6): 979-997. Doi: 10.1175/ JCLI3671.1

Gillett, N. P., Kell, T D., Jones, P. D. (2006). Regional climate impacts of the Southern Annular Mode. Geophysical Research Letters. 33 (23): Doi: 10.1029/2006GL027721

Gimeno, L., Drumond,A., Nieto, R., Trigo, R.M., Stohl,A. (2010). On the origin of continental precipitation. Geophysical Research Letters. 37 (13): Doi: 10.1029/2010GL043712

Gimeno, L., Nieto, R., Drumond, A., Castillo, R., Trigo, R. (2013). Influence of the intensification of the major oceanic moisture sources on continental precipitation. Geophysical Research Letters. 40 (7): 1443-1450, Doi: 10.1002/grl.50338

Giorgi, F. \& Francisco, R. (2000). Uncertainties in regional climate change prediction: A regional analysis of ensemble simulations with the HadCM2 coupled AOGCM. Climate Dynamics. 16 (2-3): 169-182. Doi: 10.1175/ JHM-D-11-088.1

Gochis, D. J., Brito-Castillo, L., Shuttleworth, W. J. (2007). Correlations between sea-surface temperatures and warm season streamflow in Northwest Mexico. International Journal of Climatology. 27 (7): 883-901. Doi: 10.1002/ joc. 1436

Gong, D. \& Wang, S. (1999). Definition of Antarctic oscillation index. Geophysical Research Letters. 26 (4): 459-462. Doi: 10.1029/1999GL900003

Gutzler, D. S. \& Preston, J. W. (1997). Evidence for a relationship between spring snow cover in North America and summer rainfall in New Mexico. Geophysical Research Letters. 24 (17): 2207-2210. Doi: 10.1029/97GL02099

Hales, J. E. (1972). Surges of maritime tropical air northward over the Gulf of California. Monthly Weather Review. 100 (4): 298-306. Doi: 10.1175/1520-0493(1972)100<0298:SOMT $\mathrm{AN}>2.3 . \mathrm{CO} ; 2$

Hanna, S. (1984). Applications in air pollution modeling. In Atmospheric turbulence and air pollution modelling. Springer. p. 275-310

Hidalgo, H. G., Amador, J. A., Alfaro, E. J., Quesada, B. (2013). Hydrological climate change projections for Central America. Journal of Hydrology. 495: 94-112. Doi: 10.1016/j.jhydrol.2013.05.004

Ho, M., Kiem, A., Verdon-Kidd, D. (2012). The Southern Annular Mode: A comparison of indices. Hydrology and Earth System Sciences. 16 (3): 967-982. Doi: 10.5194/ hess-16-967-2012

Huang, B., Thorne, P. W., Banzon, V. F., Boyer, T., Chepurin, G., Lawrimore, J. H., Menne, M. J., Smith, T. M., Vose, R. S., Zhang, H.-M. (2017). Extended reconstructed sea surface temperature, version 5 (ERSSTv5): Upgrades, validations, and intercomparisons. Journal of Climate. 30 (20): 8179-8205. Doi: 10.1175/JCLI-D-16-0836.1

Hurrell, J. W. (1995). Decadal trends in the North Atlantic Oscillation: Regional temperatures and precipitation. Science. 269 (5224): 676-679. Doi: 10.1126/science.269.5224.676

Hurrell, J. W. \& Van Loon, H. (1997). Decadal variations in cli-mate associated with the North Atlantic Oscillation. In Climatic change at high elevation sites. Springer, Dordrecht. p. 69-94. Doi: 10.1007/978-94-015-8905-5_4
Kalnay, E., Kanamitsu, M., Kistler, R., Collins, W., Deaven, D., Gandin, L., Iredell, M., Saha, S., White, G., Woollen, J., et al. (1996). The NCEP/NCAR 40-year reanalysis project. Bulletin of the American Meteorological Society. 77 (3): 437-472. Doi: 10.1175/1520-0477(1996)077<0437:TNYR $\mathrm{P}>2.0 . \mathrm{CO} ; 2$

Karnauskas, K. B., Seager, R., Giannini, A., Busalacchi, A. J. (2013). A simple mechanism for the climatological midsummer drought along the pacific coast of Central America. Atmósfera. 26 (2): 261-281. Doi: 10.1016/S01876236(13)71075-0

Kidston, J., Scaife, A. A., Hardiman, S. C., Mitchell, D. M., Butchart, N., Baldwin, M. P., Gray, L. J. (2015). Stratospheric influence on tropospheric jet streams, storm tracks and surface weather. Nature Geoscience. 8 (6): 433. Doi: 10.1038/ngeo2424

Kodera, K., Koide, H., Yoshimura, H. (1999). Northern Hemisphere winter circulation associated with the North Atlantic Oscillation and stratospheric polar-night jet. Geophysical Research Letters. 26 (4): 443-446. Doi: 10.1029/1999GL900016

Li, J. \& Wang, J. X. (2003). A modified zonal index and its physical sense. Geophysical Research Letters. 30 (12): 1632. Doi: 10.1029/2003GL017441

Lorenz, C. \& Kunstmann, H. (2012). The hydrological cycle in three state-of-the-art reanalyses: Intercomparison and performance analysis. Journal of Hydrometeorology. 13 (5): 1397-1420. Doi: 10.1175/JHM-D-11-088.1

Magaña, V., Amador, J. A., Medina, S. (1999). The mid-summer drought over Mexico And Central America. Journal of Climate. 12 (6): 1577-1588. Doi: 10.1175/1520-0442(1999) 012<1577:TMDOMA $>2.0 . \mathrm{CO} ; 2$

Magaña, V. O., Vázquez, J. L., Pérez, J. L., Pérez, J. B. (2003). Impact of El Niño on precipitation in Mexico. Geofísica Internacional. 42 (3): 313-330.

Maldonado, T., Rutgersson, A., Alfaro, E., Amador, J., Claremar, B. (2016). Interannual variability of the midsummer drought in Central America and the connection with sea surface temperatures. Advances in Geosciences. 42: 35-50. Doi: 10.5194/adgeo-42-35-2016

Marsh, E. J., Bruno, M. C., Fritz, S. C., Baker, P., Capriles, J. M., Hastorf, C. A. (2018). IntCal, SHCal, or a Mixed Curve? Choosing a 14 C Calibration Curve for Archaeological and Paleoenvironmental Records from Tropical South America. Radiocarbon. 60 (3): 925-940. Doi: 10.1017/RDC.2018.16

Marshall, G. J. (2003). Trends in the southern annular mode from observations and reanalyses. Journal of Climate. 16 (24): 4134-4143. Doi: 10.1175/1520-0442(2003)016<4134:TIT $\mathrm{SAM}>2.0 . \mathrm{CO} ; 2$

Maurer, E. P., Roby, N., Stewart-Frey, I. T., Bacon, C. M. (2017). Projected twenty-first-century changes in the Central American mid-summer drought using statistically downscaled climate projections. Regional Environmental Change. 17 (8): 2421-2432. Doi: 10.1007/s10113-017-1177-6

Mestas-Nuñez, A. M., Enfield, D. B., Zhang, C. (2007). Water vapor fluxes over the Intra-Americas Sea: Seasonal and interannual variability and associations with rainfall. Journal of Climate. 20 (9): 1910-1922. Doi: 10.1175/JCLI4096.1

Mosiño, A. P. \& García, E. (1966). Evaluación de la sequía intraestival en la república mexicana. Proc. Conf. Reg. Latinoamericana Unión Geogr. Int. 3: 500-516. 
Nan, S. \& Li, J. (2003). The relationship between the summer precipitation in the Yangtze River valley and the boreal spring Southern Hemisphere Annular Mode. Geophysical Research Letters. 30 (24): 2266. Doi: 10.1029/2003GL018381

Nieto, R., Castillo, R., Drumond, A., Gimeno, L. (2014a). A catalog of moisture sources for continental climatic regions. Water Resources Research. 50 (6): 5322-5328. Doi: 10.1002/2013WR013901

Nieto, R., Castillo, R., Drumond, A. (2014b). The modulation of oceanic moisture transport by the hemispheric annular modes. Frontiers in Earth Science. 2: 11. Doi: 10.3389/ feart.2014.00011

Numaguti, A. (1999). Origin and recycling processes of precipitating water over the Eurasian continent: Experiments using an atmospheric general circulation model. Journal of Geophysical Research: Atmospheres. 104 (D2): 19571972. Doi: 10.1029/1998JD200026

Poveda, G. \& Mesa, O. (1999). La corriente de chorro superficial del oeste ("del Chocó") y otras dos corrientes de chorro en Colombia: climatología y variabilidad durante las fases del ENSO. Revista Académica Colombiana de Ciencia. 23 (89): 517-528.

Rodwell, M. J., Rowell, D. P., Folland, C. K. (1999). Oceanic forcing of the wintertime North Atlantic Oscillation and European climate. Nature. 398 (6725): 320. Doi: 10.1038/18648

Sáenz, F. \& Durán-Quesada, A. M. (2015). A climatology of low level wind regimes over Central America using a weather type classification approach. Frontiers in Earth Science. 3: 15. Doi: 10.3389/feart.2015.00015

Schultz, D. M., Bracken, W. E., Bosart, L. F. (1998). Planetaryand synoptic-scale signatures associated with Central American cold surges. Monthly Weather Review. 126 (1): 5-27. Doi: 10.1175/1520-0493(1998)126<0005:PASSSA $>$ 2.0.CO; 2

Smith, T. M., Reynolds, R.W., Peterson, T. C., Lawrimore, J. (2008). Improvements to NOAA's historical merged landocean surface temperature analysis (1880-2006). Journal of Climate. 21 (10): 2283-2296. Doi: 10.1175/2007JCLI2100.1

Stahle, D. W. \& Cleaveland, M. K. (1993). Southern oscillation extremes reconstructed from tree rings of the Sierra Madre Occidental and Southern Great Plains. Journal of Climate. 6 (1): 129-140. Doi: 10.1175/1520-0442(1993)006<0129:SO ERFT>2.0.CO;2

Stohl, A., Forster, C., Frank, A., Seibert, P., Wotawa, G. (2005). The Lagrangian particle dispersion model FLEXPART version 6.2. Atmospheric Chemistry and Physics. 5 (9): 2461-2474. Doi: 10.5194/acp-5-2461-2005

Stohl, A., Hittenberger, M., Wotawa, G. (1998). Validation of the Lagrangian particle dispersion model FLEXPART against large-scale tracer experiment data. Atmospheric Environment. 32 (24): 4245-4264. Doi: 10.1016/S1352-2310(98)00184-8

Stohl, A. \& James, P. (2004). A Lagrangian analysis of the atmospheric branch of the global water cycle. Part i: Method description, validation, and demonstration for the August 2002 flooding in Central Europe. Journal of Hydrometeorology. 5 (4): 656-678. Doi: 10.1175/1525-7541(2004)005<0656:ALA OTA $>2.0 . \mathrm{CO} ; 2$

Stohl, A. \& James, P. (2005). A Lagrangian analysis of the atmospheric branch of the global water cycle. Part ii: Moisture transports between earth's ocean basins and river catchments. Journal of Hydrometeorology. 6 (6): 961-984. Doi: $10.1175 / J H M 470.1$
Stohl, A. \& Thomson, D. J. (1999). A density correction for Lagrangian particle dispersion models. Boundary-Layer Meteorology. 90 (1): 155-167. Doi: 10.1175/JHM470.1

Taylor, M. A. \& Alfaro, E. J. (2005). Climate of Central America and the Caribbean. In Encyclopedia of World Climatology. Springer. p. 183-189.

Thompson, D. W. \& Wallace, J. M. (2000). Annular modes in the extratropical circulation. Part I: Month-to-month variability. Journal of climate. 13 (5): 1000-1016. Doi: 10.1175/1520-0442(2000)013<1000:AMITEC>2.0.CO;2

Thompson, D. W. \& Wallace, J. M. (2001). Regional climate impacts of the Northern Hemisphere Annular Mode. Science. 293 (5527): 85-89. Doi: 10.1126/science. 1058958

Trenberth, K. E., Fasullo, J. T., Mackaro, J. (2011). Atmospheric moisture transports from ocean to land and global energy flows in reanalyses. Journal of Climate. 24 (18): 4907 4924. Doi: 10.1175/2011JCLI4171.1

Trenberth, K. E. \& Guillemot, C. J. (1998). Evaluation of the atmospheric moisture and hydrological cycle in the NCEP/ NCAR reanalyses. Climate Dynamics. 14 (3): 213-231. Doi: $10.1007 / \mathrm{s} 003820050219$

Uppala, S. M., Kållberg, P., Simmons, A., Andrae, U., Bechtold, V. D. C., Fiorino, M., Gibson, J., Haseler, J., Hernández, A., Kelly, G., et al. (2005). The Era-40 reanalysis. Quarterly Journal of the Royal Meteorological Society. 131 (612): 2961-3012. Doi: 10.1256/qj.04.176

Van Loon, H. \& Rogers, J. C. (1978). The seesaw in winter temperatures between Greenland and Northern Europe. Part I: General description. Monthly Weather Review. 106 (3): 296-310. Doi: 10.1175/1520-0493(1978)106<0296:TS IWTB $>2.0 . \mathrm{CO} ; 2$

Visbeck, M. (2009). A station-based Southern Annular Mode Index from 1884 to 2005. Journal of Climate. 22 (4): 940950. Doi: $10.1175 / 2008 J C L I 2260.1$

Vogelezang, D. \& Holtslag, A. (1996). Evaluation and model impacts of alternative boundary layer height formulations. Boundary-Layer Meteorology. 81 (3-4): 245-269. Doi: 10.1007/BF02430331

Wallace, J. M. \& Gutzler, D. S. (1981). Teleconnections in the geopotential height field during the Northern Hemisphere winter. Monthly Weather Review. 109 (4): 784-812. Doi: 10.1175/1520-0493(1981)109<0784:TITGHF $>2.0 . C O ; 2$

Wang, C. \& Enfield, D. B. (2001). The tropical western hemisphere warm pool. Geophysical Research Letters. 28 (8): 16351638. Doi: 10.1029/2000GL011763

Wang, C. \& Enfield, D. B. (2003). A further study of the Tropical Western Hemisphere Warm Pool. Journal of Climate. 16 (10): 1476-1493. Doi: 10.1029/2000GL011763

Wu, Z., Li, J., Wang, B., Liu, X. (2009). Can the Southern Hemisphere Annular Mode affect China winter monsoon? Journal of Geophysical Research: Atmospheres. 114(D11). Doi: 10.1029/2008JD011501

Xie, S. P., Noguchi, H., Matsumura, S. (1999). A hemisphericscale quasi-decadal oscillation and its signature in Northern Japan. Journal of the Meteorological Society of Japan. Ser. II, 77 (2): 573-582. Doi: 10.2151/jmsj1965.77.2_573

Zárate-Hernández, E. (2013). Climatología de masas invernales de aire frío que alcanzan Centroamérica y el Caribe y su relación con algunos índices árticos. Tópicos Meteorológicos y Oceanográficos. 12 (1): 35-55. 